\title{
The $C^{\infty}$-convergence of hexagonal disk packings to the Riemann map
}

\author{
by \\ ZHENG-XU HE \\ and \\ ODED SCHRAMM \\ University of California, San Diego \\ La Jolla, CA, U.S.A. \\ The Weizmann Institute of Science \\ Rehovot, Israel
}

\section{Introduction}

Let $\Omega \varsubsetneqq \mathbf{C}$ be a simply-connected domain. The Rodin-Sullivan Theorem states that a sequence of disk packings in the unit disk $U$ converges, in a well-defined sense, to a conformal map from $\Omega$ to $U$. Moreover, it is known that the first and second derivatives converge as well. Here, it is proven that for hexagonal disk packings the convergence is $C^{\infty}$. This is done by studying Möbius invariants of the disk packings that are discrete analogs of the Schwarzian derivative.

As a consequence, the first $n$ derivatives of the conformal map can be approximated by quantities which depend on the positions of the centers of some $n+1$ consecutive disks in the packing.

Suppose that $P=\left(P_{v}: v \in V\right)$ is an indexed disk packing in the plane. The nerve (or tangency graph) of $P$ is the graph $G=(E, V)$ on $V$, the indexing set of $P$, such that $[v, u] \in E$ if and only if $P_{v}$ and $P_{u}$ are tangent. The Disk Packing Theorem [10] says that given a graph $G$ which is the 1-skeleton of a topological triangulation of $\bar{U}=\{z \in \mathbf{C}$ : $|z| \leqslant 1\}$, there is some disk packing in $\bar{U}$ with nerve $G$, such that the boundary disks (i.e., those disks corresponding to the boundary vertices) are all tangent to the unit circle $\partial U$. Moreover, the disk packing is unique up to (possibly orientation-reversing) Möbius transformations of $\bar{U}$ (see, for example, [18, Chapter 13], [12], [3] or [4] for other proofs and extensions).

For each $\varepsilon>0$, let $H^{\varepsilon}$ denote the hexagonal grid with mesh $\varepsilon$. The vertices of $H^{\varepsilon}$ form the hexagonal lattice:

$$
V^{\varepsilon}=\{n \varepsilon+m \omega \varepsilon:(n, m) \in \mathbf{Z} \times \mathbf{Z}\}
$$

The first named author was supported by NSF Grant DMS 96-22068, and the second named author by NSF Grant DMS 94-03548. 

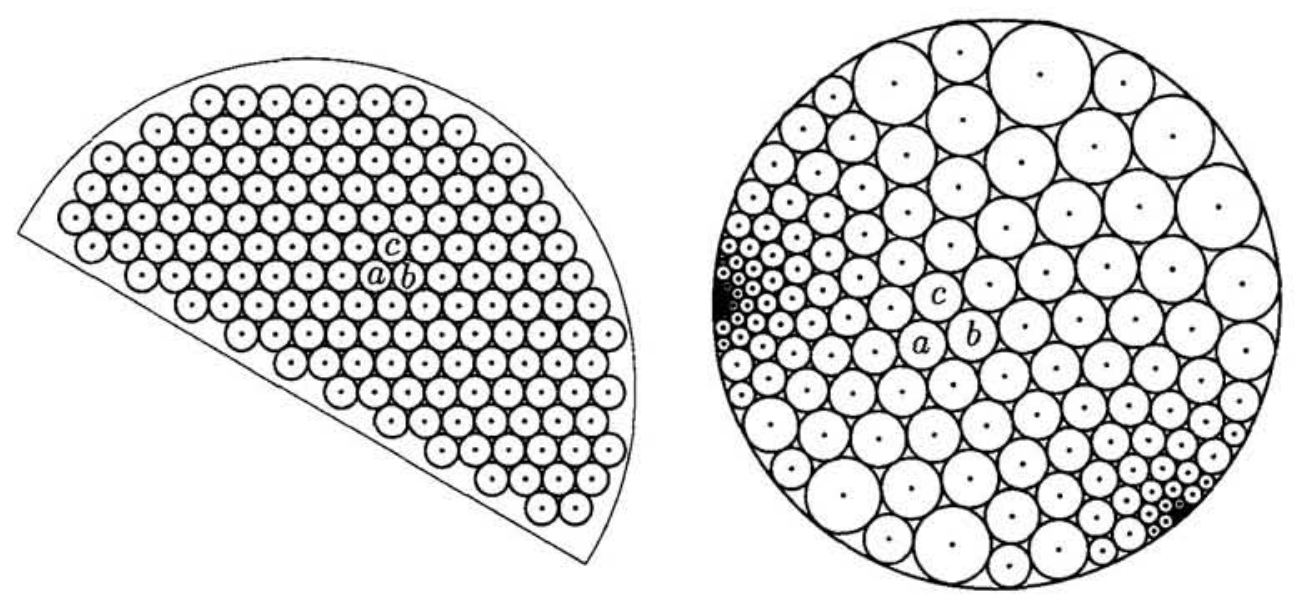

Fig. 1.1. The packings $R^{\epsilon}$ and $P^{\varepsilon}$, and the map $f^{\epsilon}$. Several points have been marked to aid in grasping the correspondence.

where $\omega$ is the cube root of -1 ,

$$
\omega=\exp \frac{1}{3} \pi i=\frac{1}{2}(\sqrt{3} i+1),
$$

and an edge connects any two vertices of $H^{\varepsilon}$ at distance $\varepsilon$.

Let $\Omega$ be a simply-connected domain in $\mathbf{C}$ with $\Omega \neq \mathbf{C}$. Then there is a subgrid $H_{\Omega}^{\varepsilon}$ of $H^{\varepsilon}$, equal to the 1-skeleton of a triangulation of a closed topological disk contained in $\Omega$ that approximates $\Omega$. Let $V_{\Omega}^{\varepsilon}$ denote the set of vertices in $H_{\Omega}^{\varepsilon}$. From the Disk Packing Theorem it follows that there is a disk packing $P^{\varepsilon}=\left(P_{v}^{\varepsilon}: v \in V_{\Omega}^{\varepsilon}\right)$ in $\bar{U}$ whose nerve is $H_{\Omega}^{\varepsilon}$ and with the property that boundary disks are tangent to $\partial U$. For each $v \in V_{\Omega}^{\varepsilon}$, let $f^{\varepsilon}(v)$ denote the center of the disk $P_{v}^{\varepsilon}$ (see Figure 1.1). Then the Rodin-Sullivan Theorem [15] tells us that, assuming that the packings $P^{\varepsilon}$ are suitably normalized (by a Möbius transformation, possibly orientation-reversing), the discrete functions $f^{\varepsilon}: V_{\Omega}^{\varepsilon} \rightarrow U$ converge "locally uniformly" in $\Omega$ to the (similarly normalized) Riemann map $f: \Omega \rightarrow U$, when $\varepsilon \rightarrow 0$.

There is a natural definition for the discrete partial derivatives of functions defined on the lattice $V_{\Omega}^{\varepsilon}$. Given discrete functions $g^{\varepsilon}: V_{\Omega}^{\varepsilon} \rightarrow \mathbf{C}$, where $\varepsilon \in(0,1)$, we say that $g^{\varepsilon}$ converge in $C^{\infty}(\Omega)$ to a smooth function $g: \Omega \rightarrow \mathbf{C}$ as $\varepsilon \rightarrow 0$, if $g^{\varepsilon}$ converge locally uniformly in $\Omega$ to $g$, and the discrete partial derivatives of $g^{\varepsilon}$ of any order converge locally uniformly to the corresponding partial derivatives of $g$. The precise definitions will be given in $\S 2$.

For each $v \in V_{\Omega}^{\varepsilon}$, let $r^{\varepsilon}(v)$ denote the radius of $P_{v}^{\varepsilon}$. Our main theorem is:

$C^{\infty}$-Convergence Theorem 1.1. The discrete functions $f^{\varepsilon}: V_{\Omega}^{\varepsilon} \rightarrow U$ converge in $C^{\infty}(\Omega)$ to the Riemann mapping $f: \Omega \rightarrow U$. The discrete functions $2 r^{\varepsilon}(v) / \varepsilon$ converge in $C^{\infty}(\Omega)$ to $\left|f^{\prime}\right|$. 
As a consequence, the derivatives of the conformal map up to order $n$ can be approximated by quantities which depend on the positions of the centers of some $n+1$ consecutive disks in the packing.

W. Thurston [19] constructed the packings $P^{\varepsilon}$, and conjectured that $f^{\varepsilon}$ converges to the Riemann mapping. The locally uniform convergence (i.e., $C^{0}$-convergence) was proved by Rodin and Sullivan [15], using a rigidity theorem for quasi-conformal deformations of Schottky groups and a length-area argument. The $C^{1}$-convergence was proved in [6], using an area estimate; and $C^{2}$-convergence in [5], using the same area estimate. The result of [5] says that the "intersticial maps" converge in $C^{2}$ to $f$. In [8] it is shown that the method of [6] works well for general disk packings with bounded valence.

Recently, the $C^{2}$-convergence of the intersticial maps has been generalized in [9] to disk packings of arbitrary combinatorial pattern, even without the assumption of bounded valence. This is done using methods of discrete extremal length and fixed point index arguments. We intend to write a paper which further simplifies [9], avoids the discrete extremal length methods, and gives estimates for the convergence rates.

Several other interesting results also appeared in [13], [14], [1] and [2]. These works may be combined together to yield an alternative proof of the $C^{1}$-convergence. We note that K. Stephenson [17] has also given a proof of the $C^{0}$-convergence using Markov chains and electrical networks "with leaks"; and recently, L. Carleson has found a different proof based on Rodin's equation [13], namely,

$$
\pi=\sum_{k=1}^{6} \tan ^{-1}\left(\sqrt{\frac{r_{k} r_{k+1}}{R\left(r_{k}+r_{k+1}+R\right)}}\right),
$$

where $r_{k}, k \in \mathbf{Z}_{6}=\mathbf{Z} / 6 \mathbf{Z}$, are the radii of six disks which surround a disk of radius $R$.

Following is a brief description of our method. First some Möbius invariants for finite hexagonal disk packings are defined and their elementary equations are derived. Similar invariants and equations were introduced [16] in the setting of circle patterns with the combinatorics of the square grid. We will then use the Möbius invariants to define (discrete) Schwarzians (or Schwarzian derivatives). Roughly, the Schwarzians are some appropriately scaled measure of the deformation of pairs of neighboring interstices from their standard positions and, as the continuous Schwarzian, they are unchanged if the packing is replaced by a Möbius image. For a regular hexagonal packing (in which all disks have identical size), the Schwarzians are tailored to be 0 . The results of [6] will be used to show that the Schwarzians of the packings $P^{\varepsilon}$ are bounded. On the other hand, from the equations satisfied by the Möbius invariants, we will show that the (discrete) Laplacian of the Schwarzians is a polynomial function in $\varepsilon$ and the Schwarzians themselves. It is then a consequence of a regularity theorem of discrete elliptic equations 
that all discrete derivatives of the Schwarzians, of any given order, are locally bounded. The $C^{\infty}$-convergence of $f^{\varepsilon}$ and $2 r^{\varepsilon} / \varepsilon$ will then follow.

It is also proved that a linear combination of the discrete Schwarzians of $P^{\varepsilon}$ converges in $C^{\infty}$ to the Schwarzian derivative of the conformal mapping. Another interesting result is that some natural "contact transformations" defined by the positions of triplets of mutually tangent disks converge in $C^{\infty}$. In fact, any reasonable, natural discrete function associated to $P^{\varepsilon}$ can be shown to be convergent in $C^{\infty}(\Omega)$.

It is possible to carry out our proof with an investigation of the radii in place of the Schwarzians, and with Rodin's equation (1.1) taking the place of the equation which the discrete Schwarzians satisfy. But perhaps some of the details become more complicated.

The rest of the paper is organized as follows. $\S 2$ introduces some discrete partial differential operators on the lattices $V^{\varepsilon}$, and defines precisely the meaning of locally uniform convergence and $C^{\infty}$-convergence. $\S 3$ gives the construction of the subgrid $H_{\Omega}^{\varepsilon}$ and fixes a normalization for $P^{\varepsilon}$, and hence for $f^{\varepsilon}$ and $f$. $\S 4$ introduces the Schwarzians, and derives the equations satisfied by them. These equations will be used in $\S 5$ to obtain the formula for the discrete Laplacian of the Schwarzians. A consequence of the formula is that if the Schwarzians are all bounded, then so are their Laplacians. In $\S 6$, we will prove that the Schwarzians are uniformly bounded in any compact subset of $\Omega$. In $\S 7$ the Lipschitz norm of a discrete derivative of a function is estimated in terms of the $L^{\infty}$. norms of the function and its discrete Laplacian. At that point, one can conclude that the Schwarzians are uniformly Lipschitz, and therefore convergent in $C^{0}(\Omega)$. In $\S 8$, local uniform bounds on the partial derivatives of any order for the Schwarzians are obtained. In $\S 9$, the contact transformations of disk patterns are defined, and it is shown that they converge in $C^{\infty}(\Omega)$. Then, in $\S 10$, the main theorem is obtained as a consequence of the $C^{\infty}$-convergence of the contact transformations. Finally, disk patterns of more general combinatorics are discussed in $\S 11$.

We thank the anonymous referee for valuable suggestions, which lead us to the current formulation of the main theorem.

\section{Preliminaries on discrete differential operators and the continuous limit of discrete functions}

We will need to introduce some discrete differential operators. For each $\varepsilon>0$, recall that the set $V^{\varepsilon}$ is the set of vertices in the hexagonal grid $H^{\varepsilon}$, i.e., the points $n \varepsilon+m \omega \varepsilon$, where $\omega=\exp \left(\frac{1}{3} \pi i\right)$, and $n, m$ are integers.

For any $k \in \mathbf{Z}_{6}$, let $L_{k}^{\varepsilon}: V^{\varepsilon} \rightarrow V^{\varepsilon}$ denote the translation

$$
L_{k}^{\varepsilon} v=v+\varepsilon \omega^{k} .
$$


Let $W \subseteq V^{\varepsilon}$ be a subset. A vertex $v \in W$ is called an interior vertex of $W$ if for each $k, k \in \mathbf{Z}_{6}$, the neighboring vertex $L_{k}^{\varepsilon} v$ is contained in $W$. Let $W^{(0)}=W$, and for each integer $l \geqslant 1$, let $W^{(l)}$ denote the set of interior vertices of $W^{(l-1)}$.

Given a function $\eta: W \rightarrow \mathbf{R}$, the (discrete) directional derivative $\partial_{k}^{\varepsilon} \eta: W^{(1)} \rightarrow \mathbf{R}$ is defined by

$$
\partial_{k}^{\varepsilon} \eta(v)=\varepsilon^{-1}\left(\eta\left(L_{k}^{\varepsilon} v\right)-\eta(v)\right)=\varepsilon^{-1}\left(\eta\left(v+\varepsilon \omega^{k}\right)-\eta(v)\right) .
$$

Let $L_{k}^{\varepsilon} \eta$ denote the function which differs from $\eta$ by the translation $L_{k}^{\varepsilon}$ :

$$
L_{k}^{\varepsilon} \eta(v)=\eta\left(L_{k}^{\varepsilon} v\right)=\eta\left(v+\varepsilon \omega^{k}\right)
$$

Then we have

$$
\partial_{k}^{\varepsilon}=\varepsilon^{-1}\left[L_{k}^{\varepsilon}-I\right]
$$

where $I \eta=\eta$.

The (discrete) Laplacian of a function $\eta: W \rightarrow \mathbf{R}$ is a function in $W^{(1)}$ defined by the formula

$$
\Delta^{\varepsilon} \eta(v)=\frac{2}{3} \varepsilon^{-2} \sum_{k \in \mathbf{Z}_{6}}\left(\eta\left(L_{k}^{\varepsilon} v\right)-\eta(v)\right)=\frac{2}{3} \varepsilon^{-2} \sum_{k \in \mathbf{Z}_{6}}\left(\eta\left(v+\varepsilon \omega^{k}\right)-\eta(v)\right) .
$$

In other words,

$$
\Delta^{\varepsilon}=\frac{2}{3} \varepsilon^{-2} \sum_{k \in \mathbf{Z}_{6}}\left(L_{k}^{\varepsilon}-I\right) .
$$

Note that the Laplacian of $x^{2}$ restricted to $V^{\varepsilon}$ is 2 . That is the reason for the factor $\frac{2}{3} \varepsilon^{-2}$.

Clearly, the operators $I, L_{j}^{\varepsilon}, \partial_{k}^{\varepsilon}$ and $\Delta^{\varepsilon}$ commute with each other.

For any function $g$ defined on a subset $W \subseteq V^{\varepsilon}$, we will use $\|g\|_{W}$ to denote its $L^{\infty}(W)$-norm:

$$
\|g\|_{W}=\sup _{v \in W}|g(v)| .
$$

For any differentiable function $G: \Omega \rightarrow \mathbf{R}$ and any $k \in \mathbf{Z}_{6}$, let $\partial_{k} G$ denote the directional derivative

$$
\partial_{k} G(z)=\lim _{t \rightarrow 0} \frac{G\left(z+t \omega^{k}\right)-G(z)}{t} .
$$

Definitions. Let $f: \Omega \rightarrow \mathbf{C}^{d}$ be some function defined in some domain $\Omega \subset \mathbf{C}$. For each $\varepsilon>0$, let $f^{\varepsilon}$ be some function defined on some set of vertices $V_{0}^{\varepsilon} \subset V^{\varepsilon}$, with values in $\mathbf{C}^{d}$. Suppose that for each $z \in \Omega$ there are some $\delta_{1}, \delta_{2}>0$ such that $\left\{v \in V^{\varepsilon}:|v-z|<\delta_{2}\right\} \subset V_{0}^{\varepsilon}$ whenever $\varepsilon \in\left(0, \delta_{1}\right)$.

If for every $z \in \Omega$ and every $\sigma>0$ there are some $\delta_{1}, \delta_{2}>0$ such that $\left|f(z)-f^{\varepsilon}(v)\right|<\sigma$, for every $\varepsilon \in\left(0, \delta_{1}\right)$ and every $v \in V^{\varepsilon}$ with $|v-z|<\delta_{2}$, then we say that $f^{\varepsilon}$ converges to $f$, locally uniformly in $\Omega$. 
Let $n \in \mathbf{N}$, and suppose that $f$ is $C^{n}$-smooth. If for every sequence $k_{1}, \ldots, k_{j} \in \mathbf{Z}_{6}$ with $j \leqslant n$ we have $\partial_{k_{j}}^{\varepsilon} \partial_{k_{j-1}}^{\varepsilon} \ldots \partial_{k_{1}}^{\varepsilon} f^{\varepsilon} \rightarrow \partial_{k_{j}} \partial_{k_{j-1}} \ldots \partial_{k_{1}} f$ locally uniformly in $\Omega$, then we say that $f^{\varepsilon}$ converges to $f$ in $C^{n}(\Omega)$. If that holds for all $n \in \mathbf{N}$, then the convergence is $C^{\infty}$.

The functions $f^{\varepsilon}$ are said to be uniformly bounded in $C^{n}(\Omega)$ provided that for every compact $K \subset \Omega$ there is some constant $C(K, n)$ such that

$$
\left\|\partial_{k_{j}}^{\varepsilon} \partial_{k_{j-1}}^{\varepsilon} \ldots \partial_{k_{1}}^{\varepsilon} f^{\varepsilon}\right\|_{K \cap V^{\varepsilon}}<C(K, n)
$$

whenever $j \leqslant n$, and $\varepsilon$ is sufficiently small. The functions $f^{\varepsilon}$ are uniformly bounded in $C^{\infty}(\Omega)$, if they are uniformly bounded in $C^{n}(\Omega)$ for every $n \in \mathbf{N}$.

Following are some simple lemmas about $C^{\infty}$-convergence of such functions.

LEMMA 2.1. Let $n$ be a positive integer. Suppose that the functions $f^{\varepsilon}$ are uniformly bounded in $C^{n}(\Omega)$. Then for every sequence of $\varepsilon \rightarrow 0$ there is a $C^{n-1}(\Omega)$-function $f$ and a subsequence of $\varepsilon \rightarrow 0$ such that $f^{\varepsilon} \rightarrow f$ in $C^{n-1}(\Omega)$ along that subsequence.

Proof. Since $f^{\varepsilon}$ are uniformly bounded in $C^{1}(\Omega)$, the standard proof of the ArzelaAscoli Theorem shows that there is a continuous function $f$ defined in $\Omega$ and a subsequence of $\varepsilon \rightarrow 0$ such that $f^{\varepsilon} \rightarrow f$ locally uniformly along that subsequence.

We apply the same argument to the discrete derivatives of the functions $f^{\varepsilon}$ with order at most $n-1$, and conclude that for some subsequence all the discrete derivatives of order at most $n-1$ of the functions $f^{\varepsilon}$ will converge locally uniformly in $\Omega$. It is then easy to verify that $f^{\varepsilon} \rightarrow f$ in $C^{n-1}(\Omega)$. The details are left to the reader.

LemMA 2.2. Suppose that $f^{\varepsilon}, g^{\varepsilon}, h^{\varepsilon}$ converge in $C^{\infty}(\Omega)$ to functions $f, g, h: \Omega \rightarrow \mathbf{C}$, and suppose that $h \neq 0$ in $\Omega$. Then the following convergences are in $C^{\infty}(\Omega)$ :

(1) $f^{\varepsilon}+g^{\varepsilon} \rightarrow f+g$,

(2) $f^{\varepsilon} g^{\varepsilon} \rightarrow f g$,

(3) $1 / h^{\varepsilon} \rightarrow 1 / h$,

(4) if $h^{\varepsilon}>0$ then $\sqrt{h^{\varepsilon}} \rightarrow \sqrt{h}$,

(5) $\left|h^{\varepsilon}\right| \rightarrow|h|$.

Proof. The local uniform convergence is obvious in each of these cases. Hence, by Lemma 2.1, it is sufficient to show that these discrete functions are uniformly bounded in $C^{\infty}(\Omega)$. Part (1) is easy. Part (2) follows from the identity

$$
\partial_{k}^{\varepsilon}\left(f^{\varepsilon} g^{\varepsilon}\right)=\left(\partial_{k}^{\varepsilon} f^{\varepsilon}\right) g^{\varepsilon}+\left(L_{k}^{\varepsilon} f^{\varepsilon}\right) \partial_{k}^{\varepsilon} g^{\varepsilon}
$$


and induction. Similarly, parts (3) and (4) follow from the identities,

$$
\begin{aligned}
\partial_{k}^{\varepsilon}\left(1 / h^{\varepsilon}\right) & =\frac{-\partial_{k}^{\varepsilon} h^{\varepsilon}}{h^{\varepsilon} L_{k}^{\varepsilon} h^{\varepsilon}}, \\
\partial_{k}^{\varepsilon} \sqrt{h^{\varepsilon}} & =\frac{\partial_{k}^{\varepsilon} h^{\varepsilon}}{\sqrt{L_{k}^{\varepsilon} h^{\varepsilon}}+\sqrt{h^{\varepsilon}}} .
\end{aligned}
$$

The details are left to the reader. The convergence of $\left|h^{\varepsilon}\right|$ is $C^{\infty}$, because $\left|h^{\varepsilon}\right|=\sqrt{h^{\varepsilon} \overline{h^{\varepsilon}}}$.

\section{The setup}

This section will describe the construction of the packings $P^{\varepsilon}$. The notations and assumptions introduced here will be adopted throughout the following.

Let $\Omega$ be a simply-connected domain in $\mathbf{C}$ with $\Omega \neq \mathbf{C}$. Fix two arbitrary distinct points $z_{0}, z_{0}^{\prime}$ in $\Omega$. We now describe a standard construction of a triangulation of a simply-connected subregion of $\Omega$ that approximates $\Omega$. For every small $\varepsilon>0$, consider the subset of vertices of $V^{\varepsilon} \cap\{z \in \mathbf{C}:|z| \leqslant 1 / \varepsilon\}$ such that their distance to $\mathbf{C}-\Omega$ is bigger than $\varepsilon$; and let $\widetilde{V}_{\Omega}^{\varepsilon}$ be the set of vertices which are either an interior vertex of this subset, or shares an edge with an interior vertex. Let $V_{\Omega}^{\varepsilon}$ be the connected component of $\tilde{V}_{\Omega}^{\varepsilon}$ which contains some vertex with distance at most $\varepsilon$ to $z_{0}$, and let $H_{\Omega}^{\varepsilon}$ be the subgraph of $H^{\varepsilon}$ spanned by the vertices in $V_{\Omega}^{\varepsilon}$. Then for small $\varepsilon, H_{\Omega}^{\varepsilon}$ is equal to the 1-skeleton of a geometric triangulation (with equilateral triangles of side length $\varepsilon$ ) of a closed topological disk contained in $\Omega$ that approximates $\Omega$.

As we stated in the introduction, it follows from the Disk Packing Theorem that there is a disk packing $P^{\varepsilon}=\left(P_{v}^{\varepsilon}: v \in V_{\Omega}^{\varepsilon}\right)$ in $\bar{U}$ whose nerve is $H_{\Omega}^{\varepsilon}$ and with the property that boundary disks are tangent to $\partial U$. We normalize $P^{\varepsilon}$ by a (possibly orientationreversing) Möbius transformation, so that (1) for a vertex $v_{0} \in V_{\Omega}^{\varepsilon}$ that is closest to $z_{0}$ the center of the disk $P_{v_{0}}^{\varepsilon}$ is 0 , (2) for a vertex $v_{0}^{\prime} \in V_{\Omega}^{\varepsilon}$ that is closest to $z_{0}^{\prime}$ the center of the disk $P_{v_{0}^{\prime}}^{\varepsilon}$ is on the positive real ray, and (3) the six disks $P_{L_{k}^{\varepsilon} v}^{\epsilon}, k=0,1, \ldots, 5$, surround $P_{v}^{\varepsilon}$ in positive circular order. Let $f^{\varepsilon}: V_{\Omega}^{\varepsilon} \rightarrow U$ be the function that maps every $v \in V_{\Omega}^{\varepsilon}$ to the center of the disk $P_{v}^{\varepsilon}$. Then the Rodin-Sullivan Theorem [15] tells us that $f^{\varepsilon}$ converges locally uniformly in $\Omega$ to the Riemann map $f: \Omega \rightarrow U$ satisfying $f\left(z_{0}\right)=0$ and $f\left(z_{0}^{\prime}\right)>0$.

Let $R^{\varepsilon}$ denote the regular hexagonal disk packing whose disks are centered at the vertices in the hexagonal grid $H^{\varepsilon}$. The disks of $R^{\varepsilon}$ all have radius $\frac{1}{2} \varepsilon$. Let $R_{\Omega}^{\varepsilon}$ be the subpacking corresponding to the subset of vertices $V_{\Omega}^{\varepsilon}$.

For any triplet of mutually tangent disks $P_{1}, P_{2}, P_{3}$ in $P^{\varepsilon}$ or $R^{\varepsilon}$, there is a disk $D$ whose boundary $\partial D$ passes through the intersection points $P_{1} \cap P_{2}, P_{2} \cap P_{3}$ and $P_{3} \cap P_{1}$. 
The disk $D$ is called a dual disk of the packing. Note that $\partial D$ is orthogonal to each of the circles $\partial P_{j}, j=1,2,3$.

\section{The discrete Schwarzians}

Let $f$ be a complex analytic function defined on a domain in the plane $\mathbf{C}$, such that $f^{\prime}(z)$ never vanishes. The Schwarzian derivative of $f$ is defined by

$$
\mathcal{S} f(z)=\left(\frac{f^{\prime \prime}(z)}{f^{\prime}(z)}\right)^{\prime}-\frac{1}{2}\left(\frac{f^{\prime \prime}(z)}{f^{\prime}(z)}\right)^{2}=\frac{f^{\prime \prime \prime}(z)}{f^{\prime}(z)}-\frac{3\left(f^{\prime \prime}(z)\right)^{2}}{2\left(f^{\prime}(z)\right)^{2}}
$$

The Schwarzian derivative of $f$ is itself a complex analytic function. It is elementary to check that for a Möbius transformation $T(z)=(a z+b) /(c z+d)$ we have $\mathcal{S}(T \circ f)(z)=$ $\mathcal{S} f(z)$. Moreover, $S f=0$ if and only if $f$ is equal to the restriction of a Möbius transformation. These and some further properties of the Schwarzian can be found in [11, $§ 1]$, for example.

In the same spirit, we will define the Möbius invariants of hexagonal disk packings, and derive their immediate equations. Analogous invariants and equations were worked out in [16] in a similar way for circle patterns based on the square grid, where applications were found to the study of global properties of immersed patterns in $\widehat{\mathbf{C}}$. Here, we will use Möbius invariants as an intermediate means in the study of the convergence problem; and the Schwarzians will be defined as some suitably scaled measure of deformation of the Möbius invariants from their regular values. As an important step, we will derive a formula for the Laplacian of the Schwarzians in the next section.

Our method will yield the same result if Rodin's equation (1.1), which the radii satisfy, is used instead. Thus, the use of Möbius invariants is not essential. We have chosen to work with the Möbius invariants as they yield relatively easier equations.

For any edge $e=[v, u]$ in $H_{\Omega}^{\varepsilon}$, we let $p_{e}$ denote the point of tangency of the two disks $P_{u}^{\varepsilon}, P_{v}^{\varepsilon}$. Let $e=[v, u]$ be some edge in $H_{\Omega}^{\varepsilon}$, let $w_{1}, w_{2}$ be the two vertices of $V^{\varepsilon}$ that neighbor with both $v$ and $u$, and suppose that $w_{1}, w_{2} \in V_{\Omega}^{\varepsilon}$. Let $T$ be a Möbius transformation that sends the tangency point $p_{e}$ to infinity. Then the two circles $\partial P_{v}, \partial P_{u}$ are mapped to lines. It follows that the four points $T\left(p_{\left[v, w_{1}\right]}\right), T\left(p_{\left[v, w_{2}\right]}\right), T\left(p_{\left[u, w_{1}\right]}\right), T\left(p_{\left[u, w_{2}\right]}\right)$ are at the corners of a rectangle, see Figure 4.1.

Since $T$ is well-determined up to post-composing by a similarity, the aspect ratio of the rectangle,

$$
\frac{\left|T\left(p_{\left[v, w_{1}\right]}\right)-T\left(p_{\left[v, w_{2}\right]}\right)\right|}{\left|T\left(p_{\left[v, w_{1}\right]}\right)-T\left(p_{\left[u, w_{1}\right]}\right)\right|}
$$




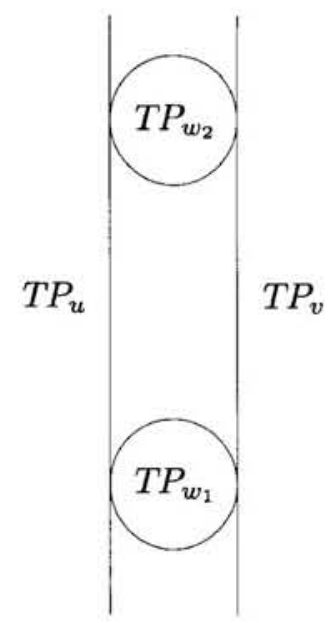

Fig. 4.1. The configuration after applying $T$.

is independent of the choice of $T$. It also does not change if we modify $P^{\varepsilon}$ by a Möbius transformation. Set $s_{e}$ to be this aspect ratio divided by $\sqrt{3}$,

$$
s(e)=\frac{\left|T\left(p_{\left[v, w_{1}\right]}\right)-T\left(p_{\left[v, w_{2}\right]}\right)\right|}{\sqrt{3}\left|T\left(p_{\left[v, w_{1}\right]}\right)-T\left(p_{\left[u, w_{1}\right]}\right)\right|},
$$

and let

$$
h(e)=\varepsilon^{-2}(s(e)-1)
$$

be called the (discrete) Schwarzian derivative, or Schwarzian of $P^{\varepsilon}$ at $e$. The factor $1 / \sqrt{3}$ in (4.2) is justified by the fact that when the disks $P_{u}, P_{v}, P_{w_{1}}, P_{w_{2}}$ are all the same size, we get $s(e)=1$ and $h(e)=0$. The factor $\varepsilon^{-2}$ is reasonable, because of the behavior of the Schwarzian derivative under rescaling, namely, $(\mathcal{S} g)(z)=\varepsilon^{2}(\mathcal{S} f)(\varepsilon z)$ when $g(z)=f(\varepsilon z)$. (This is also justified by the estimate of $\S 6$.)

For a vertex $v$ in $V^{\varepsilon}$, denote $e_{k}(v)=\left[v, L_{k}^{\varepsilon}(v)\right]$. See Figure 4.2. Let $s_{k}, h_{k}:\left(V_{\Omega}^{\varepsilon}\right)^{(1)} \rightarrow \mathbf{R}$ be defined by $s_{k}(v)=s\left(e_{k}(v)\right)$ and $h_{k}(v)=h\left(e_{k}(v)\right)$. Clearly, $s_{k}(v)=s_{k+3}\left(L_{k} v\right)$ and $h_{k}(v)=h_{k+3}\left(L_{k} v\right)$.

LeMma 4.1. Let $v$ be an interior vertex in $V_{\Omega}^{\varepsilon}$. Then,

$$
s_{k}(v)+s_{k+2}(v)+s_{k+4}(v)=3 s_{k}(v) s_{k+1}(v) s_{k+2}(v)
$$

is valid for any $k \in \mathbf{Z}_{6}$.

Although we will not prove it here, the equations (4.4) are sufficient to guarantee that a positive function $s$ on the edges of $H^{\varepsilon}$ corresponds to an (immersed) hexagonal circle pattern (cf. [16]). 


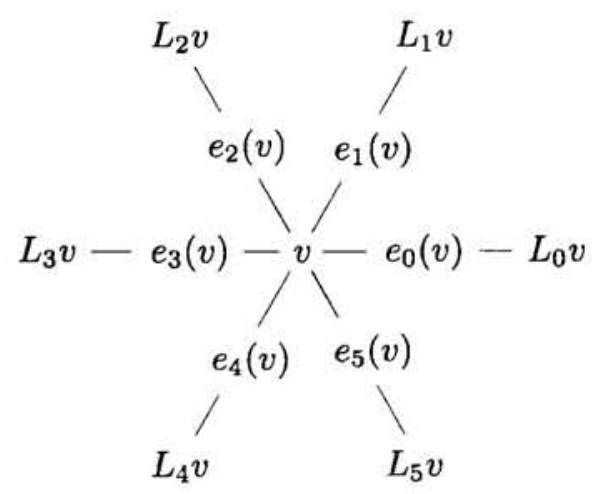

Fig. 4.2. The edges around a vertex.

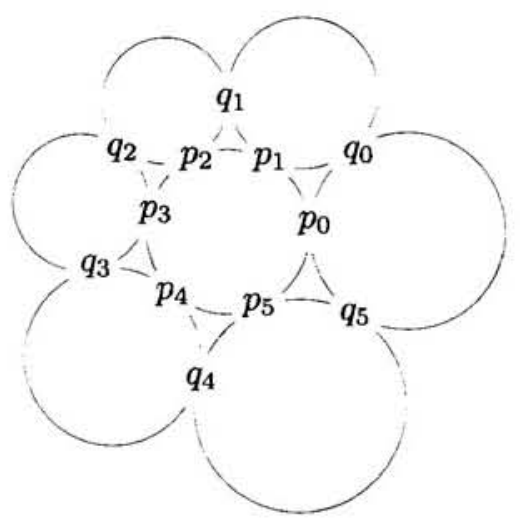

Fig. 4.3. The special points of a flower.

Proof. For any $k \in \mathbf{Z}_{6}$, let $p_{k}$ be the point of tangency $P_{v}^{\varepsilon} \cap P_{L_{k}^{\varepsilon} v}^{\varepsilon}$, and let $q_{k}$ be the point in $P_{L_{k}^{\varepsilon} v}^{\varepsilon} \cap P_{L_{k+1}^{\varepsilon} v}^{\varepsilon}$. See Figure 4.3. There is no loss of generality, because the packing $P^{\varepsilon}$ may be reflected about a line.

Let $m_{k}=m_{k}(v)$ be the orientation-preserving Möbius transformation that takes $p_{k}, p_{k-1}, q_{k-1}$ to $\infty, 0,1$, respectively. Then, by the definition of the $s_{k}$ 's,

$$
\begin{aligned}
m_{k}(v)\left(p_{k+1}\right) & =-\sqrt{3} s_{k} i, \\
m_{k}(v)\left(q_{k}\right) & =1-\sqrt{3} s_{k} i, \\
m_{k}(v)\left(p_{k}\right) & =\infty
\end{aligned}
$$

Consequently, setting $M_{k}=m_{k+1} \circ m_{k}^{-1}$, we get

$$
\begin{aligned}
M_{k}\left(-\sqrt{3} s_{k} i\right) & =\infty \\
M_{k}\left(1-\sqrt{3} s_{k} i\right) & =1, \\
M_{k}(\infty) & =0 .
\end{aligned}
$$


Therefore,

$$
M_{k}=\left(\begin{array}{cc}
0 & i \\
i & -\sqrt{3} s_{k}
\end{array}\right),
$$

where the usual matrix notation for Möbius transformations is used. Note that the composition $M_{5} \circ M_{4} \circ M_{3} \circ M_{2} \circ M_{1} \circ M_{0}$ is the identity. Hence, $M_{5} \circ M_{4} \circ M_{3}=M_{0}^{-1} \circ M_{1}^{-1} \circ M_{2}^{-1}$, which evaluates to

$$
\left(\begin{array}{cc}
\sqrt{3} s_{4} & 3 i s_{3} s_{4}-i \\
3 i s_{4} s_{5}-i & \sqrt{3}\left(s_{3}+s_{5}-3 s_{3} s_{4} s_{5}\right)
\end{array}\right)=\left(\begin{array}{cc}
\sqrt{3}\left(s_{0}+s_{2}-3 s_{0} s_{1} s_{2}\right) & i-3 i s_{0} s_{1} \\
i-3 i s_{1} s_{2} & \sqrt{3} s_{1}
\end{array}\right) .
$$

This is an equality of Möbius transformations, and is therefore valid up to a scalar factor. However, both sides have determinant 1 , because the matrix in (4.5) has determinant 1 , and therefore (4.6) is valid up to sign. From the upper left entries, we get

$$
\pm s_{4}=s_{0}+s_{2}-3 s_{0} s_{1} s_{2} \text {. }
$$

Because $s_{4}$ is never zero, and since the set of configurations of 6 disks in a 'flower' around a given disk is connected, the sign in (4.7) does not depend on the configuration. When all circles have the same radius, $s_{k}=1$, so the correct sign is minus. This proves (4.4) for $k=0$. The equations for the other values of $k$ are valid by symmetry.

\section{The Laplacian of the discrete Schwarzians}

In this section, we will use the equations of the $s_{k}$ 's of $\S 4$ to obtain the equations for the $h_{k}$ 's, and these will be used to show that $\Delta^{\varepsilon} h_{k}(v)$ is equal to a polynomial in $\varepsilon, h_{j_{0}}(v), L_{j_{2}} h_{j_{1}}(v) ; j_{0}, j_{1}, j_{2} \in \mathbf{Z}_{6}$. We consider only $\varepsilon$ in the range $(0,1)$, and therefore, if all $h_{k}$ 's are uniformly bounded in a vertex subset $W$, then so are the $\Delta^{\varepsilon} h_{k}$ 's in the set $W^{(1)}$.

We substitute $s_{k}(v)=1+\varepsilon^{2} h_{k}(v)$ in equation (4.4), simplify, and get

$$
\begin{aligned}
h_{k}(v)+h_{k+2}(v)+h_{k+4}(v)= & 3 h_{k}(v)+3 h_{k+1}(v)+3 h_{k+2}(v) \\
& +3 \varepsilon^{2}\left(h_{k}(v) h_{k+1}(v)+h_{k+1}(v) h_{k+2}(v)+h_{k+2}(v) h_{k}(v)\right) \\
& +3 \varepsilon^{4} h_{k}(v) h_{k+1}(v) h_{k+2}(v) .
\end{aligned}
$$

Set

$$
\begin{aligned}
\Psi_{k+1}(v)=- & \left(h_{k}(v) h_{k+1}(v)+h_{k+1}(v) h_{k+2}(v)+h_{k+2}(v) h_{k}(v)\right) \\
& -\varepsilon^{2} h_{k}(v) h_{k+1}(v) h_{k+2}(v) .
\end{aligned}
$$

Then equation (5.1) becomes

$$
2 h_{k}(v)+3 h_{k+1}(v)+2 h_{k+2}(v)-h_{k+4}(v)=3 \varepsilon^{2} \Psi_{k+1}(v) .
$$

Replace $k$ by $k+2$, multiply by 2 , add to (5.3), and get

$$
3 h_{k+1}(v)+6 h_{k+2}(v)+6 h_{k+3}(v)+3 h_{k+4}(v)=3 \varepsilon^{2} \Psi_{k+1}(v)+6 \varepsilon^{2} \Psi_{k+3}(v) .
$$




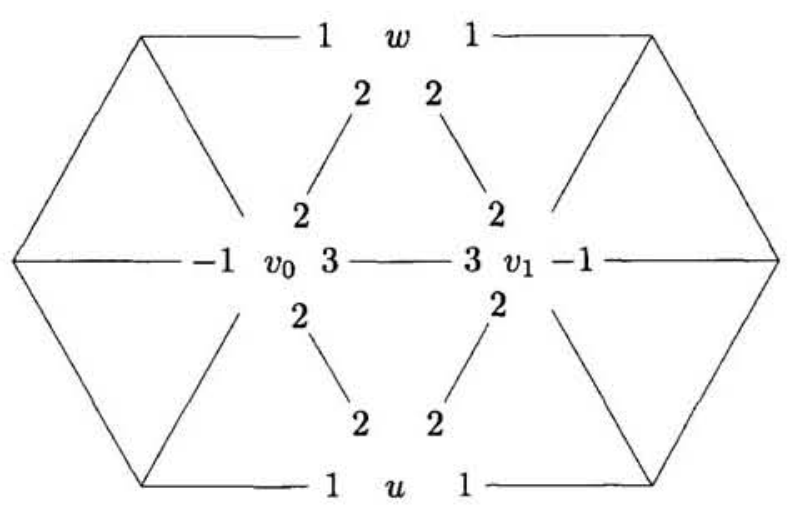

Fig. 5.1. The numbers mark the coefficients of the $h$-values in the identities (5.5) through (5.8).

LEMMA 5.1. $\Delta^{\varepsilon} h_{k}(v)$ is equal to a polynomial in the variables $\varepsilon, h_{j_{0}}(v), L_{j_{1}}^{\varepsilon} h_{j_{2}}(v)$; $j_{0}, j_{1}, j_{2} \in \mathbf{Z}_{6}$. More specifically,

$$
\Delta^{\varepsilon} h_{k}=\frac{2}{3} L_{k+1}^{\varepsilon} \Psi_{k+3}+\frac{4}{3} L_{k+1}^{\varepsilon} \Psi_{k+5}+\frac{2}{3} L_{k+5}^{\varepsilon} \Psi_{k}+\frac{4}{3} L_{k+5}^{\varepsilon} \Psi_{k+2}-2 L_{k}^{\varepsilon} \Psi_{k+3}-2 \Psi_{k} .
$$

It is quite fortunate that $\Delta^{\varepsilon} h_{k}(v)$ is a polynomial in $\varepsilon, h_{j_{0}}(v), L_{j_{1}}^{\varepsilon} h_{j_{2}}(v) ; j_{0}, j_{1}, j_{2} \in \mathbf{Z}_{6}$, since that simplifies many of the arguments that follow. However, the proof could be made to work if $\Delta^{\varepsilon} h_{k}(v)$ was only a $C^{\infty}$-function of these variables. This might be important for generalizing the methods of this work to other settings. See $\S 11$ for further discussion.

Proof. We work on the case $k=0$. Fix some $v_{0} \in\left(V_{\Omega}^{\varepsilon}\right)^{(2)}$. Let $v_{1}=L_{0} v_{0}, u=L_{5} v_{0}$ and $w=L_{1} v_{0}$. To understand the following computation, the reader is advised to consult Figure 5.1.

Apply equation (5.4) with $v$ replaced by $u$ and $k$ replaced by 5 , then use the relations $h_{k}(v)=h_{k+3}\left(L_{k} v\right)$, to get (after division by 3 )

$$
h_{0}(u)+2 h_{4}\left(v_{1}\right)+2 h_{5}\left(v_{0}\right)+h_{0}\left(L_{4} v_{0}\right)=\varepsilon^{2} \Psi_{0}(u)+2 \varepsilon^{2} \Psi_{2}(u) .
$$

Similarly, apply equation (5.4) with $v$ replaced by $w$ and $k$ replaced by 2, and obtain

$$
h_{0}\left(L_{2} v_{0}\right)+2 h_{1}\left(v_{0}\right)+2 h_{2}\left(v_{1}\right)+h_{0}(w)=\varepsilon^{2} \Psi_{3}(w)+2 \varepsilon^{2} \Psi_{5}(w) .
$$

The substitution $v_{0}$ for $v$ and 5 for $k$ in (5.3) gives

$$
2 h_{5}\left(v_{0}\right)+3 h_{0}\left(v_{0}\right)+2 h_{1}\left(v_{0}\right)-h_{0}\left(L_{3} v_{0}\right)=3 \varepsilon^{2} \Psi_{0}\left(v_{0}\right) .
$$

Similarly, the substitution $v_{1}$ for $v$ and 2 for $k$ in (5.3) gives

$$
2 h_{2}\left(v_{1}\right)+3 h_{0}\left(v_{0}\right)+2 h_{4}\left(v_{1}\right)-h_{0}\left(v_{1}\right)=3 \varepsilon^{2} \Psi_{3}\left(v_{1}\right) \text {. }
$$


Now subtract the sum of equations (5.7) and (5.8) from the sum of equations (5.5) and (5.6), and get

$$
\frac{3}{2} \varepsilon^{2} \Delta^{\varepsilon} h_{0}\left(v_{0}\right)=\varepsilon^{2} \Psi_{3}(w)+2 \varepsilon^{2} \Psi_{5}(w)+\varepsilon^{2} \Psi_{0}(u)+2 \varepsilon^{2} \Psi_{2}(u)-3 \varepsilon^{2} \Psi_{3}\left(v_{1}\right)-3 \varepsilon^{2} \Psi_{0}\left(v_{0}\right) .
$$

This proves the lemma for the case $k=0$. For other values of $k$ the lemma is also valid, by symmetry.

\section{The discrete Schwarzians are bounded}

In this section, we will recall the result of [6] which essentially says that the discrete Schwarzian derivative is uniformly bounded in any compact subset of the domain $\Omega$. The bound is independent of $\varepsilon$. Precisely, we have the following lemma.

LEMMA 6.1. Let $v_{0}$ be a vertex of $V_{\Omega}^{\varepsilon}$, and suppose that the distance $\delta$ from $v_{0}$ to $\{z \in \mathbf{C}:|z|>1 / \varepsilon\}-\Omega$ is greater than $2 \varepsilon$. Then

$$
\left|h_{k}\left(v_{0}\right)\right|=\varepsilon^{-2}\left|s_{k}\left(v_{0}\right)-1\right| \leqslant C, \quad k \in \mathbf{Z}_{6},
$$

for some constant $C=C(\delta)$, which depends only on $\delta$.

The proof is quite similar to the proof of Lemma 1.5 in [5]. The boundedness of the Schwarzians is equivalent to the boundedness of the third order derivatives of $f^{\varepsilon}$. It is an open problem whether the above lemma can be proved directly using Rodin's equation (1.1) for the radii, or from the formula for the Laplacian of the Schwarzians.

Proof. By [6], there is a homeomorphism $g^{\varepsilon}$ from the carrier of $R_{\Omega}^{\varepsilon}$ onto the carrier of $P^{\varepsilon}$ with the following properties:

(1) For each $v \in V_{\Omega}^{\varepsilon}$, the image of the disk $R_{v}^{\varepsilon}$ under $g^{\varepsilon}$ is the corresponding disk $P_{v}^{\varepsilon}$.

(2) The restriction of $g^{\varepsilon}$ to a dual disk of the packing $R_{\Omega}^{\varepsilon}$ is equal to a Möbius transformation.

(3) There is a universal constant $C_{1}>1$ such that for each $v \in\left(V_{\Omega}^{\varepsilon}\right)^{(2)}$, the map $g^{\varepsilon}$ restricted to $R_{v}^{\varepsilon}$ is $C_{1}$-quasiconformal.

(4) For each $v \in\left(V_{\Omega}^{\varepsilon}\right)^{(2)}$, there is a constant $C_{2}=C_{2}(\delta(v))>0$, which depends only on the distance $\delta(v)$ from $v$ to $\{z \in \mathbf{C}:|z|>1 / \varepsilon\}-\Omega$, such that the area of the subset of $R_{v}^{\varepsilon}$ where $g^{\varepsilon}$ fails to be conformal is bounded by $C_{2} \varepsilon^{4}$. (Note that the area of $R_{v}^{\varepsilon}$ is $\frac{1}{4} \pi \varepsilon^{2}$.)

Consider the restriction of $g^{\varepsilon}$ to $R_{v_{0}}^{\varepsilon}$. Let $\widetilde{D}_{k}$ be the dual disk bounded by the circle which passes through the tangency points of pairs of the disks $R_{v_{0}}^{\varepsilon}, R_{L_{k}^{\varepsilon} v_{0}}^{\varepsilon}$ and $R_{L_{k+1}^{\varepsilon} v_{0}}^{\varepsilon}$. See Figure 6.1.

Let $z_{1}, z_{2}, z_{3}, z_{4}$ be a quadruple of points on the circle $\partial R_{v_{0}}^{\varepsilon}$ which are: (1) sufficiently spaced out, say, $\left|z_{j_{1}}-z_{j_{2}}\right| \geqslant \frac{1}{100} \varepsilon$, for any $j_{1} \neq j_{2} ;(2)$ away from the points of tangency 


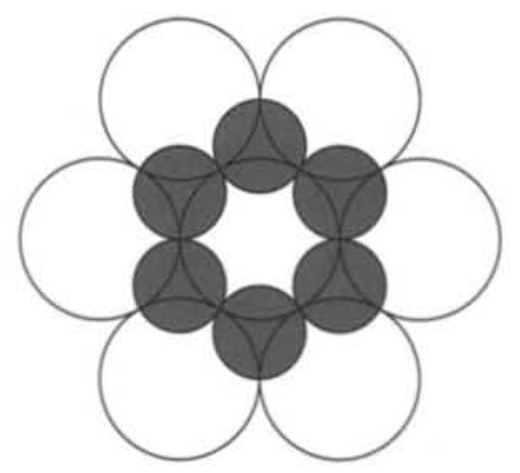

Fig. 6.1. The six dual disks.

$\left\{w_{k}\right\}=R_{v_{0}}^{\varepsilon} \cap R_{L_{k} v_{0}}^{\varepsilon}$, say, $\left|z_{j}-w_{k}\right| \geqslant \frac{1}{100} \varepsilon$; and (3) cyclicly ordered in the counter-clockwise direction. We claim that for any such quadruple,

$$
\left|\left[g^{\varepsilon}\left(z_{1}\right), g^{\varepsilon}\left(z_{2}\right) ; g^{\varepsilon}\left(z_{3}\right), g^{\varepsilon}\left(z_{4}\right)\right]-\left[z_{1}, z_{2} ; z_{3}, z_{4}\right]\right| \leqslant C_{3} \varepsilon^{2},
$$

where $[\cdot, \cdot ; \cdot, \cdot]$ denotes the cross ratio, and $C_{3}$ depends only on $\delta$.

In fact, there are positive numbers $m$ and $m^{*}$ such that the quadrilaterals $\left(R_{v_{0}}^{\varepsilon} ; z_{1}, z_{2}, z_{3}, z_{4}\right)$ and $\left(g^{\varepsilon}\left(R_{v_{0}}^{\varepsilon}\right) ; g^{\varepsilon}\left(z_{1}\right), g^{\varepsilon}\left(z_{2}\right), g^{\varepsilon}\left(z_{3}\right), g^{\varepsilon}\left(z_{4}\right)\right)$ are conformally homeomorphic to the standard rectangles

$$
\left(Q_{m}=[0, m] \times[0,1] ;(0,0),(m, 0),(m, 1),(0,1)\right)
$$

and

$$
\left(Q_{m^{*}}=\left[0, m^{*}\right] \times[0,1] ;(0,0),\left(m^{*}, 0\right),\left(m^{*}, 1\right),(0,1)\right),
$$

respectively. The map $g^{\varepsilon}$ will then be translated to a $C_{1}$-quasiconformal map $F^{\varepsilon}$ between the standard rectangles

$$
\left(Q_{m} ;(0,0),(m, 0),(m, 1),(0,1)\right) \text { and }\left(Q_{m^{*}} ;(0,0),\left(m^{*}, 0\right),\left(m^{*}, 1\right),(0,1)\right) .
$$

The relations $\left|z_{j_{1}}-z_{j_{2}}\right| \geqslant \frac{1}{100} \varepsilon, j_{1} \neq j_{2}$, imply that $m$ and $m^{*} \in\left[m / C_{1}, m C_{1}\right]$ are bounded from above and below by some universal positive constants. On the other hand, since $\left|z_{j}-w_{k}\right| \geqslant \frac{1}{100} \varepsilon$, by property (2) above we deduce that $g^{\varepsilon}$ is conformal in the $\frac{1}{200} \varepsilon$ neighborhood of the points $z_{j}, 1 \leqslant j \leqslant 4$. Outside the $\frac{1}{200} \varepsilon$-neighborhoods of the $z_{j}$ 's, the conformal homeomorphism from $R^{\varepsilon}$ onto $Q_{m}$ is clearly Lipschitz, with Lipschitz constant bounded by $C_{4} \varepsilon^{-1}$, where $C_{4}$ is a universal constant. Thus, using property (4), it follows that $F^{\varepsilon}$ is conformal except on a subset of area bounded by $C_{4}^{2} C_{2} \varepsilon^{2}$. It then 
follows by a standard Grötsch argument (compare $[6, \S 2]$ ) that $\left|m^{*} / m-1\right| \leqslant C_{5} \varepsilon^{2}$, where $C_{5}$ depends on $C_{1}$ and $C_{2}$. Then (6.2) follows as $m$ and $m^{*}$ are related to the cross ratios $\left[z_{1}, z_{2} ; z_{3}, z_{4}\right]$ and $\left[g^{\varepsilon}\left(z_{1}\right), g^{\varepsilon}\left(z_{2}\right) ; g^{\varepsilon}\left(z_{3}\right), g^{\varepsilon}\left(z_{4}\right)\right]$, respectively, by the same smooth function.

Let $T_{k}$ be the Möbius transformation which agrees with $g^{\varepsilon}$ on $\widetilde{D}_{k}$. Since a Möbius transformation is uniquely determined by its values at three points, it follows from (6.2) that for any $k \in \mathbf{Z}_{6}$,

$$
\left|\varepsilon^{-1} T_{k}^{-1} \circ T_{k-1}(z)-\varepsilon^{-1} z\right| \leqslant C_{6} \varepsilon^{2}, \quad \forall z \in R_{v_{0}}^{\varepsilon}
$$

where $C_{6}$ depends only on $C_{3}$, and consequently, on $\delta$.

It is then elementary to check that (6.3) implies that $\left|s_{k}\left(v_{0}\right)-1\right| \leqslant \varepsilon^{2} C_{7}$, with $C_{7}$ depending only on $\delta$, which gives (6.1).

\section{Regularity of solutions of discrete elliptic equations}

Regularity Lemma 7.1. Let $W$ be a subset of $V^{\varepsilon}$, let $v_{0} \in W^{(1)}$, and let $\delta$ be the Euclidean distance from $v_{0}$ to $V^{\varepsilon}-W$. Let $\eta: W \rightarrow \mathbf{R}$ be any function. Then

$$
\delta\left|\partial_{k}^{\varepsilon} \eta\left(v_{0}\right)\right| \leqslant 7\|\eta\|_{W}+\frac{1}{2} \delta^{2}\left\|\Delta^{\varepsilon} \eta\right\|_{W^{(1)}}
$$

holds for any $k \in \mathbf{Z}_{6}$.

This lemma is known in the continuous setting, and may certainly also be known in the discrete setting. The estimate is not sharp. For lack of a good reference, we include a proof.

Proof. Note that both sides of (7.1) are scale invariant; i.e., if we define $\hat{\eta}: a^{-1} W \rightarrow \mathbf{R}$ by $\hat{\eta}(v)=\eta(a v)$, where $a>0$, then (7.1) for $\eta$ is equivalent to (7.1) for $\hat{\eta}$ at $a^{-1} v_{0}$. Hence, we assume with no loss of generality that $\delta=1$. Also assume for convenience, $k=3, v_{0}=\varepsilon$. Again, there is no loss of generality.

Let $R(x+i y)=(\varepsilon-x)+i y$ be the reflection in the line $x=\frac{1}{2} \varepsilon$, and set

$$
g(v)=\eta(v)-\eta(R v)
$$

As $L_{3}^{\varepsilon} v_{0}=v_{0}+(-1) \varepsilon=0=R v_{0}$, what we need to prove is that

$$
\left|g\left(v_{0}\right)\right|=|g(\varepsilon)| \leqslant \varepsilon\left(7\|\eta\|_{W}+\frac{1}{2}\left\|\Delta^{\varepsilon} \eta\right\|_{W^{(1)}}\right)
$$

This is obvious if $2 \leqslant 7 \varepsilon$, so assume $7 \varepsilon<2$. Also assume, with no loss of generality, that $g(\varepsilon)>0$. 
Let

$$
\begin{aligned}
& W_{0}=\left\{x+i y \in V^{\varepsilon}: \sqrt{\left(x-\frac{1}{2} \varepsilon\right)^{2}+y^{2}}<1-\frac{1}{2} \varepsilon\right\}, \\
& W_{1}=\left\{x+i y \in W_{0}: x \geqslant \frac{1}{2} \varepsilon\right\} .
\end{aligned}
$$

Since $W_{0}=R\left(W_{0}\right)$ is contained in $W$, the function $g=\eta-\eta \circ R$ is well-defined in $W_{0}$. Denote $\tilde{x}=x-\frac{1}{2} \varepsilon$. Consider the "comparison" function $\hat{g}: W_{0} \rightarrow \mathbf{R}$ defined by

$$
\hat{g}(x+i y)=\hat{g}\left(\tilde{x}+\frac{1}{2} \varepsilon+i y\right)=\left(7\left(\tilde{x}^{2}+y^{2}\right)+14\left(\tilde{x}-\tilde{x}^{2}\right)\right)\|\eta\|_{W}+\left(\tilde{x}-\tilde{x}^{2}\right)\left\|\Delta^{\varepsilon} \eta\right\|_{W^{(1)}},
$$

and note that $\hat{g} \geqslant 0$ on $W_{1}$.

At any interior vertex of $W_{0}$, we have $\Delta^{\varepsilon}(g-\hat{g}) \geqslant 0$, because

$$
\Delta^{\varepsilon} g(v)=\Delta^{\varepsilon} \eta(v)-\Delta^{\varepsilon} \eta(R v) \geqslant-2\left\|\Delta^{\varepsilon} \eta\right\|_{W^{(1)}},
$$

and $\Delta^{\varepsilon} \hat{g}(v)=-2\left\|\Delta^{\varepsilon} \eta\right\|_{W^{(1)}}$.

In particular, $\Delta^{\varepsilon}(g-\hat{g}) \geqslant 0$ at any interior vertex of $W_{1}$. Then it is elementary to see (from (2.4)) that the maximum of $g-\hat{g}$ on $W_{1}$ is attained at some boundary vertex, say $v_{*}=x_{*}+i y_{*}$, of $W_{1}$ (the maximum principle). We claim that

$$
(g-\hat{g})\left(v_{*}\right) \leqslant \frac{7}{4} \varepsilon^{2}\|\eta\|_{W}+\frac{1}{4} \varepsilon^{2}\left\|\Delta^{\varepsilon} \eta\right\|_{W^{(1)}} .
$$

Clearly, the set of boundary vertices of $W_{1}$ is contained in the union of the subsets

$$
\begin{aligned}
& B_{1}=\left\{x+i y \in W_{1}: x=\frac{1}{2} \varepsilon\right\}, \\
& B_{2}=\left\{x+i y \in W_{1}: x=\varepsilon\right\}, \\
& B_{3}=\left\{x+i y \in W_{1}: \sqrt{\left(x-\frac{1}{2} \varepsilon\right)^{2}+y^{2}} \geqslant 1-\frac{3}{2} \varepsilon\right\} .
\end{aligned}
$$

If $v_{*}=\frac{1}{2} \varepsilon+i y_{*} \in B_{1}$, then $R v_{*}=v_{*}, g\left(v_{*}\right)=0$, and $g\left(v_{*}\right)=0 \leqslant \hat{g}\left(v_{*}\right)$, hence $(g-\hat{g})\left(v_{*}\right) \leqslant 0$. If $v_{*} \in B_{3}$ then

$$
\begin{aligned}
\hat{g}\left(v_{*}\right) & \geqslant 7\left(\left(x_{*}-\frac{1}{2} \varepsilon\right)^{2}+y_{*}^{2}\right)\|\eta\|_{W} \geqslant 7\left(1-\frac{3}{2} \varepsilon\right)^{2}\|\eta\|_{W} \\
& \geqslant 7\left(1-\frac{3}{7}\right)^{2}\|\eta\|_{W} \geqslant 2\|\eta\|_{W} \geqslant\left|g\left(v_{*}\right)\right|
\end{aligned}
$$

and therefore again $(g-\hat{g})\left(v_{*}\right) \leqslant 0$.

Now let $v_{*}=\varepsilon+i y_{*} \in B_{2}-B_{3}$. In this case, $\varepsilon+i y_{*}$ is an interior vertex of $W_{0}$. Since $\Delta^{\varepsilon}(g-\hat{g})\left(v_{*}\right) \geqslant 0$, and $(g-\hat{g})\left(\varepsilon+i y_{*}\right)$ is the maximum of $g-\hat{g}$ in $W_{1}$, and $i y_{*}$ is the only neighbor of $v_{*}=\varepsilon+i y_{*}$ outside of $W_{1}$, it follows that

$$
(g-\hat{g})\left(i y_{*}\right) \geqslant(g-\hat{g})\left(\varepsilon+i y_{*}\right) .
$$


As $g\left(i y_{*}\right)=g\left(R\left(\varepsilon+i y_{*}\right)\right)=-g\left(\varepsilon+i y_{*}\right)$, we deduce that $\hat{g}\left(\varepsilon+i y_{*}\right)-\hat{g}\left(i y_{*}\right) \geqslant 2 g\left(\varepsilon+i y_{*}\right)$, and then

$$
(g-\hat{g})\left(\varepsilon+i y_{*}\right) \leqslant-\frac{1}{2}\left(\hat{g}\left(\varepsilon+i y_{*}\right)+\hat{g}\left(i y_{*}\right)\right) \leqslant \frac{7}{4} \varepsilon^{2}\|\eta\|_{W}+\frac{1}{4} \varepsilon^{2}\left\|\Delta^{\varepsilon} \eta\right\|_{W^{(1)}} .
$$

This proves estimate (7.4).

Since $(g-\hat{g})\left(v_{*}\right)$ is the maximum of $g-\hat{g}$ in $W_{1}$ and since $\varepsilon \in W_{1}$, we have $(g-\hat{g})\left(v_{*}\right) \geqslant$ $(g-\hat{g})(\varepsilon)$, and then by (7.4),

$$
\begin{aligned}
|g(\varepsilon)| & =g(\varepsilon)=\hat{g}(\varepsilon)+(g-\hat{g})(\varepsilon) \leqslant \hat{g}(\varepsilon)+(g-\hat{g})\left(v_{*}\right) \\
& \leqslant \hat{g}(\varepsilon)+\frac{7}{4} \varepsilon^{2}\|\eta\|_{W}+\frac{1}{4} \varepsilon^{2}\left\|\Delta^{\varepsilon} \eta\right\|_{W^{(1)}}=14\left(\frac{1}{2} \varepsilon\right)\|\eta\|_{W}+\frac{1}{2} \varepsilon\left\|\Delta^{\varepsilon} \eta\right\|_{W^{(1)}},
\end{aligned}
$$

which implies (7.2), and proves the lemma.

\section{The discrete Schwarzians converge}

In this section, we show that for some sequence of $\varepsilon \rightarrow 0$, the Schwarzians $h_{k}$ converge in $C^{\infty}$. In a later section, the limit will be identified, and it will follow that the limit exists even without restricting to a subsequence.

We shall sometimes write $h_{k}^{\varepsilon}$ for $h_{k}$, to stress the dependence on $\varepsilon$. From Lemma 6.1 we know that the functions $h_{k}^{\varepsilon}$ are bounded on compact subsets of $\Omega$, with a bound independent of $\varepsilon$. Lemma 5.1 then shows that the functions $\Delta^{\varepsilon} h_{k}^{\varepsilon}$ are also uniformly bounded on compact subsets of $\Omega$. Applying Lemma 7.1, we see that also $\partial_{j}^{\varepsilon} h_{k}^{\varepsilon}$ have such a bound. It then follows, by Lemma 2.1, that for some sequence of $\varepsilon$ tending to 0 the continuous limits

$$
\mathcal{H}_{k}=\lim _{\varepsilon \rightarrow 0} h_{k}^{\varepsilon}
$$

exist, and are locally Lipschitz functions on $\Omega$.

Note for future use that

$$
\mathcal{H}_{k+3}=\mathcal{H}_{k}
$$

because $h_{k}^{\varepsilon}(v)=h_{k+3}^{\varepsilon}\left(L_{k}^{\varepsilon} v\right)$. Using this together with (5.4) gives

$$
\mathcal{H}_{0}+\mathcal{H}_{1}+\mathcal{H}_{2}=0
$$

For each $\delta>0$ let $V_{\delta}^{\varepsilon}$ denote the set of vertices of $V^{\varepsilon}$ whose Euclidean distance to $\{z \in \mathbf{C}:|z|>1 / \varepsilon\}-\Omega$ is at least $\delta$.

LemMA 8.1. Let $\delta>0$, and let $n$ be an integer. Then there are constants $C=C(n, \delta)$, $\alpha=\alpha(n, \delta)>0$ such that

$$
\left\|\partial_{k_{n}}^{\varepsilon} \partial_{k_{n-1}}^{\varepsilon} \ldots \partial_{k_{1}}^{\varepsilon} h_{k_{0}}^{\varepsilon}\right\|_{V_{\delta}^{\varepsilon}}<C
$$


holds whenever $\varepsilon<\alpha$, and $k_{0}, k_{1}, \ldots, k_{n} \in \mathbf{Z}_{6}$. In other words, the functions $h_{k}^{\varepsilon}$ are uniformly bounded in $C^{\infty}(\Omega)$.

Proof. The proof will be inductive. The case $n=0$ is handled by Lemma 6.1. So assume that $n>0$, and that the lemma holds for $0,1,2, \ldots, n-1$. Set

$$
g=\partial_{k_{n-1}}^{\varepsilon} \ldots \partial_{k_{1}}^{\varepsilon} h_{k_{0}}^{\varepsilon}
$$

Then,

$$
\Delta^{\varepsilon} g=\Delta^{\varepsilon} \partial_{k_{n-1}}^{\varepsilon} \ldots \partial_{k_{1}}^{\varepsilon} h_{k_{0}}^{\varepsilon}=\partial_{k_{n-1}}^{\varepsilon} \ldots \partial_{k_{1}}^{\varepsilon} \Delta^{\varepsilon} h_{k_{0}}^{\varepsilon} .
$$

From Lemma 5.1 it now follows that $\Delta^{\varepsilon} g$ is a linear combination of functions of the form

$$
\partial_{k_{n-1}}^{\varepsilon} \ldots \partial_{k_{1}}^{\varepsilon} \Lambda \Psi_{j_{1}}
$$

with $\Lambda=L_{j_{2}}^{\varepsilon}$ or $\Lambda=I$, the identity operator. Recall, from (5.2), that $\Psi_{j}$ is a polynomial in $\varepsilon$ and the $h_{k}$ 's. Also note the rule for discrete differentiation of a product,

$$
\partial_{k}^{\varepsilon}\left(\psi_{1} \psi_{2}\right)=\left(\partial_{k}^{\varepsilon} \psi_{1}\right) \psi_{2}+\left(L_{k}^{\varepsilon} \psi_{1}\right) \partial_{k}^{\varepsilon} \psi_{2}
$$

which is easy to verify. From this rule, it follows that $\partial_{j_{2}}^{\varepsilon} \Psi_{j_{1}}$ is a polynomial in $\varepsilon$ and the $\Lambda h_{j}$ 's, where $\Lambda$ ranges over the operators $I, \partial_{j_{2}}^{\varepsilon}, L_{j_{2}}$. By induction, it follows that

$$
\partial_{k_{n-1}}^{\varepsilon} \ldots \partial_{k_{1}}^{\varepsilon} \Psi_{k}
$$

is a polynomial in $\varepsilon$ and expressions of the form

$$
L_{j_{m}}^{\varepsilon} \ldots L_{j_{s+1}}^{\epsilon} \partial_{j_{s}}^{\varepsilon} \ldots \partial_{j_{1}}^{\varepsilon} h_{j_{0}}
$$

where $m \leqslant n-1$. If $v \in V_{\delta / 2}^{\varepsilon}, m \leqslant n$, and $4 n \varepsilon<\delta$, then $v^{\prime}=L_{j_{m}}^{\varepsilon} \ldots L_{j_{s+1}}^{\varepsilon}(v)$ is in $v \in V_{\delta / 4}^{\varepsilon}$. Therefore, the inductive hypothesis with $v^{\prime}=L_{j_{m}}^{\varepsilon} \ldots L_{j_{s+1}}^{\varepsilon}(v), n^{\prime}=s, \delta^{\prime}=\frac{1}{4} \delta$ applies, and provides a bound for (8.5) at $v$. Since $\Delta^{\varepsilon} g$ is a polynomial in $\varepsilon$ and the expressions of the form (8.5) with $m \leqslant n, s \leqslant n-1$, it follows that there is a constant $C_{1}=C_{1}(\delta)$ such that

$$
\left\|\Delta^{\varepsilon} g(v)\right\|_{V_{\delta / 2}^{\varepsilon}} \leqslant C_{1}
$$

Because $|g|$ is also bounded on $V_{\delta / 2}^{\varepsilon}$, the Regularity Lemma 7.1 provides a bound for $\left|\partial_{k_{n}}^{\varepsilon} g\right|$, on $V_{\delta}^{\varepsilon}$, which completes the induction step and the proof. 
Corollary 8.2. $h_{k}^{\varepsilon} \rightarrow \mathcal{H}_{k}$ in $C^{\infty}(\Omega)$ as $\varepsilon \rightarrow 0$.

Proof. It follows from Lemma 8.1 and Lemma 2.1 that this is true for some sequence of $\varepsilon \rightarrow 0$. The general statement will follow later, when (8.1) is proved in full generality.

Remark 8.3. It can be shown that the rate of convergence in Corollary 8.2 depends only on $n$ and $\delta$; i.e., there is a function $A(n, \delta, \varepsilon)$ such that

$$
\left\|\partial_{k_{n}}^{\varepsilon} \ldots \partial_{k_{1}}^{\varepsilon} h_{k}^{\varepsilon}-\partial_{k_{n}} \ldots \partial_{k_{1}} \mathcal{H}_{k}\right\|_{V_{\delta}^{\epsilon}}<A(n, \delta, \varepsilon) \quad \text { and } \quad A(n, \delta, \varepsilon) \rightarrow 0 \text { as } \varepsilon \rightarrow 0
$$

The following proposition will not be used below, but is interesting in itself, and would probably prove useful for studying the rate of convergence in Corollary 8.2.

Proposition 8.4. Let $\delta>0$, let $k \in \mathbf{Z}_{6}$, and suppose that $2 \varepsilon<\delta$. Then

$$
\left\|\Delta^{\varepsilon} h_{k}\right\|_{V_{\delta}^{e}} \leqslant \varepsilon^{2} C^{*},
$$

where $C^{*}=C^{*}(\delta)$ depends only on $\delta$.

We will need the following notation. Write

$$
a \stackrel{\varepsilon^{n}}{=} b
$$

when $a-b$ is a polynomial in $\varepsilon$, and in the functions $h_{k}$ and their discrete derivatives of arbitrary order, which is divisible by $\varepsilon^{n}$. We also use a similar notation for discrete operators. For example, the relations $L_{k-1}^{\varepsilon} L_{k+1}^{\varepsilon}=L_{k}^{\varepsilon}$ and $L_{j}^{\varepsilon}=I+\varepsilon \partial_{j}^{\varepsilon}$ give

$$
\partial_{k-1}^{\varepsilon}+\partial_{k+1}^{\varepsilon} \stackrel{\varepsilon}{=} \partial_{k}^{\varepsilon} \text {. }
$$

Proof. We will prove that $\Delta^{\varepsilon} h_{k} \stackrel{\varepsilon^{2}}{=} 0$. This actually proves more than the proposition; it shows that the discrete derivatives of $\Delta^{\varepsilon} h_{k}$ are also $O\left(\varepsilon^{2}\right)$.

Use Lemma 5.1 and the identities $L_{j}^{\varepsilon}=I+\varepsilon \partial_{j}^{\varepsilon}$ to write

$$
\begin{aligned}
\frac{3}{2} \Delta^{\varepsilon} h_{k}= & L_{k+1}^{\varepsilon} \Psi_{k+3}+2 L_{k+1}^{\varepsilon} \Psi_{k+5}+L_{k+5}^{\varepsilon} \Psi_{k}+2 L_{k+5}^{\varepsilon} \Psi_{k+2}-3 L_{k}^{\varepsilon} \Psi_{k+3}-3 \Psi_{k} \\
= & \Psi_{k+3}+2 \Psi_{k+5}+\Psi_{k}+2 \Psi_{k+2}-3 \Psi_{k+3}-3 \Psi_{k} \\
& \quad+\varepsilon\left(\partial_{k+1}^{\varepsilon} \Psi_{k+3}+2 \partial_{k+1}^{\varepsilon} \Psi_{k+5}+\partial_{k+5}^{\varepsilon} \Psi_{k}+2 \partial_{k+5}^{\varepsilon} \Psi_{k+2}-3 \partial_{k}^{\varepsilon} \Psi_{k+3}\right) \\
=- & 2 \Psi_{k}+2 \Psi_{k+2}-2 \Psi_{k+3}+2 \Psi_{k+5} \\
& \quad+\varepsilon\left(\partial_{k+1}^{\varepsilon} \Psi_{k+3}+2 \partial_{k+1}^{\varepsilon} \Psi_{k+5}+\partial_{k+5}^{\varepsilon} \Psi_{k}+2 \partial_{k+5}^{\varepsilon} \Psi_{k+2}-3 \partial_{k}^{\varepsilon} \Psi_{k+3}\right) .
\end{aligned}
$$

Recall that (5.3) gives $\Psi_{j}=\frac{1}{3} \varepsilon^{-2}\left(2 h_{j-1}+3 h_{j}+2 h_{j+1}-h_{j+3}\right)$. This relation implies that $-\Psi_{k}+\Psi_{k+2}-\Psi_{k+3}+\Psi_{k+5}=0$. Therefore, (8.7) reduces to

$$
\frac{3}{2} \Delta^{\varepsilon} h_{k}=\varepsilon\left(\partial_{k+1}^{\varepsilon} \Psi_{k+3}+2 \partial_{k+1}^{\varepsilon} \Psi_{k+5}+\partial_{k+5}^{\varepsilon} \Psi_{k}+2 \partial_{k+5}^{\varepsilon} \Psi_{k+2}-3 \partial_{k}^{\varepsilon} \Psi_{k+3}\right) .
$$


The relation $h_{k+2}=L_{k+2}^{\varepsilon} h_{k-1}=h_{k-1}+\varepsilon \partial_{k+2}^{\varepsilon} h_{k-1}$, and the definition (5.2) of $\Psi$ give

$$
\begin{aligned}
\Psi_{k+1} & \stackrel{\varepsilon^{2}}{=}-h_{k} h_{k+1}-h_{k+1} h_{k+2}-h_{k+2} h_{k} \\
& =-h_{k} h_{k+1}-h_{k+1}\left(h_{k-1}+\varepsilon \partial_{k+2}^{\varepsilon} h_{k-1}\right)-\left(h_{k-1}+\varepsilon \partial_{k+2}^{\varepsilon} h_{k-1}\right) h_{k} \\
& \stackrel{\varepsilon}{=}-h_{k} h_{k+1}-h_{k+1} h_{k-1}-h_{k-1} h_{k} \stackrel{\varepsilon^{2}}{=} \Psi_{k} .
\end{aligned}
$$

This means that

$$
\Psi_{j} \stackrel{\varepsilon}{=} \Psi_{k}, \quad \text { for each } j, k \in \mathbf{Z}_{6},
$$

which also implies that $\partial_{m}^{\varepsilon} \Psi_{j} \stackrel{\varepsilon}{=} \partial_{m}^{\varepsilon} \Psi_{k}$, for $m, j, k \in \mathbf{Z}_{6}$. So (8.8) further reduces to

$$
\frac{3}{2} \Delta^{\varepsilon} h_{k} \stackrel{\varepsilon^{2}}{=} \varepsilon\left(3 \partial_{k+1}^{\varepsilon}+3 \partial_{k+5}^{\varepsilon}-3 \partial_{k}^{\varepsilon}\right) \Psi_{k} .
$$

Now the relation (8.6) shows that $\frac{3}{2} \Delta^{\varepsilon} h_{k} \stackrel{\varepsilon^{2}}{=} 0$, and the proof is complete.

Remark 8.5. Another interesting identity is

$$
\frac{3}{2} \varepsilon \partial_{j}^{\varepsilon} h_{j+3} \stackrel{\varepsilon^{2}}{=} h_{j}+h_{j+2}+h_{j+4} .
$$

This follows from (5.3) with $k=j+2$.

\section{The contact transformations}

Let $v \in V_{\Omega}^{\varepsilon}$ be some interior vertex, and let $k \in \mathbf{Z}_{6}$. Define the contact transformation $Z_{k}^{\varepsilon}=Z_{k}^{\varepsilon}(v)$ to be the Möbius transformation that takes each of the three points $\varepsilon, \varepsilon \omega^{2}, \varepsilon \omega^{4}$ to the three points in $P_{v}^{\varepsilon} \cap P_{L_{k}^{\varepsilon} v}^{\varepsilon}, P_{L_{k}^{\varepsilon} v}^{\varepsilon} \cap P_{L_{k+1}^{\varepsilon}}^{\varepsilon}$ and $P_{v}^{\varepsilon} \cap P_{L_{k+1}^{\epsilon}}^{\varepsilon}$, respectively.

We now derive expressions for the discrete derivatives of $Z_{k}^{\varepsilon}(v)$ with respect to $v$. These will enable us to show that the Möbius transformation $Z_{k}^{\varepsilon}$ converge $C^{\infty}$ as $\varepsilon \rightarrow 0$.

Let $R$ be the rotation $R(z)=\omega^{2} z$. It is clear that

$$
Z_{k+2}^{\varepsilon}\left(L_{k}^{\varepsilon} v\right)=Z_{k}^{\varepsilon}(v) \cdot R
$$

Let $A=A^{\varepsilon}$ be the Möbius transformation taking $\varepsilon, \varepsilon \omega^{2}, \varepsilon \omega^{4}$ to $0,1, \infty$, respectively. Using the Möbius transformations $m_{k}(v)$ from $\S 4$, we may write

$$
Z_{k}^{\varepsilon}(v)=m_{k+1}(v)^{-1} \cdot A .
$$

With the notation $M_{k}=m_{k+1} \cdot m_{k}^{-1}$, we then have

$$
Z_{k-1}^{\varepsilon}=m_{k}^{-1} \cdot A=m_{k+1}^{-1} \cdot A \cdot A^{-1} \cdot M_{k} \cdot A=Z_{k}^{\varepsilon} \cdot A^{-1} \cdot M_{k} \cdot A .
$$


Set

$$
Q_{k}=A^{-1} \cdot M_{k} \cdot A
$$

Then the above relation can be abbreviated

$$
Z_{k-1}^{\varepsilon}=Z_{k}^{\varepsilon} \cdot Q_{k}
$$

Equations (9.1) and (9.3) will allow us to write the expression for $Z_{j}^{\varepsilon}\left(L_{k}^{\varepsilon} v\right)$ in terms of $Z_{k}^{\varepsilon}(v)$ and the transition matrices $Q_{k}, R$ :

$$
\begin{aligned}
L_{k}^{\varepsilon} Z_{k}^{\varepsilon} & =L_{k}^{\varepsilon}\left(Z_{k+2}^{\varepsilon} \cdot Q_{k+2} \cdot Q_{k+1}\right)=L_{k}^{\varepsilon} Z_{k+2}^{\varepsilon} \cdot L_{k}^{\varepsilon} Q_{k+2} \cdot L_{k}^{\varepsilon} Q_{k+1} \\
& =Z_{k}^{\varepsilon} \cdot R \cdot L_{k}^{\varepsilon} Q_{k+2} \cdot L_{k}^{\varepsilon} Q_{k+1} .
\end{aligned}
$$

Matrix representations of $R$ and $A$ are

$$
R=\left(\begin{array}{cc}
\omega & 0 \\
0 & \omega^{-1}
\end{array}\right), \quad A=\left(\begin{array}{cc}
-\omega^{2} & \varepsilon \omega^{2} \\
1 & \varepsilon \omega
\end{array}\right)
$$

and the expression (4.5) for $M_{k}$ can be written

$$
M_{k}=\left(\begin{array}{cc}
0 & i \\
i & -\sqrt{3} s_{k}
\end{array}\right)=\left(\begin{array}{cc}
0 & i \\
i & -\sqrt{3}\left(1+\varepsilon^{2} h_{k}\right)
\end{array}\right) .
$$

This gives the following expression for $Q_{k}$ :

$$
Q_{k}=\left(\begin{array}{cc}
i \omega\left(1+\varepsilon^{2} h_{k}\right) & i \omega^{2} \varepsilon\left(2+\varepsilon^{2} h_{k}\right) \\
\varepsilon i \omega h_{k} & i \omega^{2}\left(1+\varepsilon^{2} h_{k}\right)
\end{array}\right)
$$

Observe that $Q_{k}$ is polynomial in $\varepsilon$ and $h_{k}$. A direct computation gives the leading terms for $R \cdot L_{k}^{\varepsilon} Q_{k+2} \cdot L_{k}^{\varepsilon} Q_{k+1}$ as

$$
R \cdot L_{k}^{\varepsilon} Q_{k+2} \cdot L_{k}^{\varepsilon} Q_{k+1}=I+\varepsilon\left(\begin{array}{cc}
0 & 2 i \sqrt{3} \\
\omega^{5} L_{k}^{\varepsilon} h_{k+1}+\omega^{4} L_{k}^{\varepsilon} h_{k+2} & 0
\end{array}\right)+\varepsilon^{2} O(1)
$$

where $I$ is the identity matrix, and $O(1)$ denotes some matrix that is polynomial in $\varepsilon, L_{k}^{\varepsilon} h_{k+1}, L_{k}^{\varepsilon} h_{k+2}$. The equations (9.4) and (9.6) give an expression for the discrete derivative $\partial_{k}^{\varepsilon} Z_{k}^{\varepsilon}$ as

$$
\partial_{k}^{\varepsilon} Z_{k}^{\varepsilon}=Z_{k}^{\varepsilon} \cdot\left(\begin{array}{cc}
0 & 2 i \sqrt{3} \\
\omega^{5} L_{k}^{\varepsilon} h_{k+1}+\omega^{4} L_{k}^{\varepsilon} h_{k+2} & 0
\end{array}\right)+\varepsilon Z_{k}^{\varepsilon} \cdot O(1)
$$

An entirely similar computation gives

$$
\partial_{k-2}^{\varepsilon} Z_{k}^{\varepsilon}=Z_{k}^{\varepsilon} \cdot\left(\begin{array}{cc}
0 & 3-\sqrt{3} i \\
\omega h_{k-1}+h_{k} & 0
\end{array}\right)+\varepsilon Z_{k}^{\varepsilon} \cdot O(1) .
$$


(Of course, the $O(1)$-matrix here is not necessarily the same as in (9.7).) Because of the relations $\partial_{k+3}^{\varepsilon}=-\partial_{k}^{\varepsilon} L_{k+3}^{\varepsilon}$ and $\partial_{k-1}^{\varepsilon}=\partial_{k}^{\varepsilon}+\partial_{k-2}^{\varepsilon} L_{k}^{\varepsilon}$, the expressions for all the derivatives $\partial_{j}^{\varepsilon} Z_{k}^{\varepsilon}, j \in \mathbf{Z}_{6}$, can be obtained from (9.7) and (9.8).

Recall that $z_{0}$ denotes an arbitrary but fixed point in $\Omega$. For each small $\varepsilon>0$ let $v_{0}=v_{0}^{\varepsilon}$ be some vertex in $V_{\Omega}^{\varepsilon}$ which is closest to $z_{0}$. Let

$$
\widehat{Z}_{k}^{\varepsilon}(v)=Z_{k}^{\varepsilon}\left(v_{0}\right)^{-1} Z_{k}^{\varepsilon}(v)
$$

Then we have from (9.7) and (9.8) and the similar expressions for the other $\partial_{j}^{\varepsilon} Z_{k}^{\varepsilon}$,

$$
L_{j}^{\varepsilon} \widehat{Z}_{k}^{\varepsilon}=\widehat{Z}_{k}^{\varepsilon} \cdot(I+\varepsilon O(1)) .
$$

Since the absolute values of the entries in $(I+\varepsilon O(1))^{n}$ are bounded by $e^{C n}$, for some constant $C$, and since $\widehat{Z}_{k}^{\varepsilon}\left(v_{0}\right)=I$, it follows that the matrices $\widehat{Z}_{k}^{\varepsilon}$ are bounded in compact subsets of $\Omega$, independently of $\varepsilon$. From the similar relations for $Z_{k}^{\varepsilon}$, we have for each $j \in \mathbf{Z}_{6}$,

$$
\partial_{j}^{\varepsilon} \widehat{Z}_{k}^{\varepsilon}=\widehat{Z}_{k}^{\varepsilon} \cdot O(1)
$$

Note that the $O(1)$-term is bounded in $C^{\infty}(\Omega)$. Therefore, repeated differentiation of (9.9) shows that $\widehat{Z}_{k}^{\varepsilon}$ is bounded in $C^{\infty}(\Omega)$ uniformly in $\varepsilon$. By Lemma 2.1, it follows that for some sequence of $\varepsilon \rightarrow 0$, the limit

$$
\widehat{\mathcal{Z}}_{k}=\lim _{\varepsilon \rightarrow 0} \widehat{Z}_{k}^{\varepsilon}
$$

exists, and the convergence is $C^{\infty}(\Omega)$.

Multiply equation (9.7) on the left by $Z_{k}^{\varepsilon}\left(v_{0}\right)^{-1}$, and take a limit as $\varepsilon \rightarrow 0$, to obtain

$$
\partial_{k} \widehat{\mathcal{Z}}_{k}=\widehat{\mathcal{Z}}_{k} \cdot\left(\begin{array}{cc}
0 & 2 i \sqrt{3} \\
\omega^{5} \mathcal{H}_{k+1}+\omega^{4} \mathcal{H}_{k+2} & 0
\end{array}\right) .
$$

Applying a similar procedure to (9.8) gives

$$
\partial_{k-2} \widehat{\mathcal{Z}}_{k}=\widehat{\mathcal{Z}}_{k} \cdot\left(\begin{array}{cc}
0 & 3-\sqrt{3} i \\
\omega \mathcal{H}_{k-1}+\mathcal{H}_{k} & 0
\end{array}\right) .
$$

The identities (9.10), (9.11), (8.2) and (8.3) now imply

$$
\partial_{k-2} \widehat{\mathcal{Z}}_{k}=\omega^{-2} \partial_{k} \widehat{\mathcal{Z}}_{k},
$$

which shows that $\widehat{\mathcal{Z}}_{k}(z)$ is a (matrix-valued) analytic function of $z$.

Observe that the equations (9.10) and (9.11) show that the determinant of $\widehat{\mathcal{Z}}_{k}(z)$ is constant in $\Omega$. At $z_{0}$ this determinant is 1 . Therefore, $\widehat{\mathcal{Z}}_{k}(z)$ is a Möbius transformation for every $z \in \Omega$. 
The next step is to show that there is also convergence of a subsequence of $Z_{k}^{\varepsilon}$ as $\varepsilon \rightarrow 0$. For this, all that is needed is to show that the transformations $Z_{k}^{\varepsilon}\left(v_{0}\right)$ are bounded independently of $\varepsilon$. Let $\widehat{\mathcal{Z}}_{k}(z)(w)$ denote the image of $w$ under the Möbius transfromation $\widehat{\mathcal{Z}}_{k}(z)$. Since $\widehat{\mathcal{Z}}_{k}\left(z_{0}\right)=I$, we find from $(9.10)$,

$$
\left.\frac{\partial}{\partial z}\right|_{z=0} \widehat{\mathcal{Z}}_{0}(z)(0)=2 i \sqrt{3} \neq 0 .
$$

It follows that for a sufficiently small $\alpha>0$, the three points $w_{0}=\widehat{\mathcal{Z}}_{0}\left(z_{0}\right)(0), w_{1}=$ $\widehat{\mathcal{Z}}_{0}\left(z_{0}-\alpha\right)(0), w_{2}=\widehat{\mathcal{Z}}_{0}\left(z_{0}+\alpha\right)(0)$ are all distinct. Set $z_{1}=z_{0}-\alpha$ and $z_{2}=z_{0}+\alpha$.

From the Rodin-Sullivan Theorem and the definition of $Z_{0}^{\varepsilon}$, it follows that

$$
\lim _{\varepsilon \rightarrow 0} Z_{0}^{\varepsilon}(z)(\varepsilon)=f(z)
$$

where $f$ is the Riemann map $f: \Omega \rightarrow U$. For each $\varepsilon>0$, let $v_{1}, v_{2}$ be vertices of $V^{\varepsilon}$ closest to $z_{1}, z_{2}$, respectively. The Möbius transformation $Z_{0}^{\varepsilon}\left(v_{0}\right)$ takes each of the three points $\widehat{Z}_{0}^{\varepsilon}\left(v_{0}\right)(\varepsilon), \widehat{Z}_{0}^{\varepsilon}\left(v_{1}\right)(\varepsilon), \widehat{Z}_{0}^{\varepsilon}\left(v_{2}\right)(\varepsilon)$ to a point close to $f\left(z_{0}\right), f\left(z_{1}\right), f\left(z_{2}\right)$, respectively. The former triplet of points are close to $w_{0}, w_{1}, w_{2}$, respectively. Consequently, $\lim _{\varepsilon \rightarrow 0} Z_{0}^{\varepsilon}\left(v_{0}\right)$ exists, and is the Möbius transformation that takes $w_{0}, w_{1}, w_{2}$ to $f\left(z_{0}\right), f\left(z_{1}\right), f\left(z_{2}\right)$, respectively. It is easy to see that the same must hold for the other transformations $Z_{k}^{\varepsilon}\left(v_{0}\right)$. Consequently, there is $C^{\infty}$-convergence,

$$
\mathcal{Z}_{k}=\lim _{\varepsilon \rightarrow 0} Z_{k}^{\varepsilon}
$$

along some subsequence of $\varepsilon \rightarrow 0$.

TheOREM 9.1. Let $f$ be the Riemann map from $\Omega$ to the unit disk $U$. Then

$$
\mathcal{S}(f)=4\left(\mathcal{H}_{0}+\omega^{4} \mathcal{H}_{1}+\omega^{2} \mathcal{H}_{2}\right)
$$

Using (8.3), this also gives

$$
6 \mathcal{H}_{k}=\operatorname{Re}\left(\omega^{2 k} \mathcal{S}(f)\right),
$$

for $k \in \mathbf{Z}_{6}$. The theorem then implies that Corollary 8.2 and (8.1) are valid for every sequence of $\varepsilon \rightarrow 0$, not just for one particular sequence.

Proof. Write

$$
\mathcal{Z}_{0}(z)=\left(\begin{array}{ll}
a(z) & b(z) \\
c(z) & d(z)
\end{array}\right) .
$$

It follows from $f(z)=\mathcal{Z}_{0}(z)(0)$ that $f(z)=b(z) / d(z)$. Equation (9.10) is also valid for the matrix $\mathcal{Z}_{k}$, and therefore $b(z)$ and $d(z)$ both satisfy the differential equation

$$
w^{\prime \prime}=2 i \sqrt{3}\left(\omega^{5} \mathcal{H}_{1}+\omega^{4} \mathcal{H}_{2}\right) w .
$$


Consequently, we have $\left(b^{\prime} d-b d^{\prime}\right)^{\prime}=0$, and therefore $b^{\prime} d-b d^{\prime}$ is a constant, say $\alpha$. Hence, $(b / d)^{\prime}=\alpha / d^{2}$. Using the fact that $d$ satisfies (9.13), the definition (4.1) of $\mathcal{S}(f)$, and the identity (8.3), it is easy now to verify that $\mathcal{S}(f)=\mathcal{S}(b / d)$ is the same as the right-hand side of (9.12).

\section{0. $C^{\infty}$-convergence of disk packings}

Let the situation be as described in $§ 3$. For each $v \in V_{\Omega}^{\varepsilon}$, let $g^{\varepsilon}(v)$ be the contact point of $P_{v}^{\varepsilon}$ with $P_{L_{0}^{\varepsilon} v}^{\varepsilon}$. We recall that $f^{\varepsilon}(v)$ denotes the center of the disk $P_{v}^{\varepsilon}$, and $r^{\varepsilon}(v)$ the radius of $P_{v}^{\varepsilon}$. Clearly, Theorem 1.1 follows from the following theorem.

THEOREM 10.1. In the setting of $\S 3, f^{\varepsilon}$ and $g^{\varepsilon}$ converge to the conformal map $f: \Omega \rightarrow U$ in $C^{\infty}(\Omega)$, and $2 r^{\varepsilon} / \varepsilon$ converges in $C^{\infty}(\Omega)$ to $\left|f^{\prime}\right|$.

Proof. Let us start with $g^{\varepsilon}$. We have $g^{\varepsilon}(v)=Z_{0}^{\varepsilon}(v)(\varepsilon)$. Write $Z_{0}^{\varepsilon}(v)$ as a matrix,

$$
Z_{0}^{\varepsilon}(v)=\left(\begin{array}{ll}
a^{\varepsilon}(v) & b^{\varepsilon}(v) \\
c^{\varepsilon}(v) & d^{\varepsilon}(v)
\end{array}\right)
$$

Similarly, let the entries of $\mathcal{Z}_{0}(z)$ be $a(z), b(z), c(z), d(z)$. Then $\varepsilon c^{\varepsilon}+d^{\varepsilon}$ converges $C^{\infty}$ to $d$ and $\varepsilon a^{\varepsilon}+b^{\varepsilon}$ converges $C^{\infty}$ to $b$. Note that $d$ is nonzero in $\Omega$, because $b(z) / d(z)=$ $\mathcal{Z}_{0}(z)(0)=f(z)$, and the determinant of $\mathcal{Z}_{0}(z)$ is nonzero. Consequently, by (2) and (3) of Lemma 2.2, it follows that $\left(\varepsilon a^{\varepsilon}+b^{\varepsilon}\right) /\left(\varepsilon c^{\varepsilon}+d^{\varepsilon}\right) \rightarrow b / d$ in $C^{\infty}$. But that is the same as $g^{\varepsilon} \rightarrow f$.

Let $c_{1}$ be the circle that contains the three points $\varepsilon, \varepsilon \omega^{2}, \varepsilon \omega^{4}$. Because $Z_{0}^{\varepsilon}(v)$ maps the three points $\varepsilon, \varepsilon \omega^{2}, \varepsilon \omega^{4}$ to $P_{v}^{\varepsilon} \cap P_{L_{0}^{\varepsilon} v}^{\varepsilon}, P_{L_{1}^{\varepsilon} v}^{\varepsilon} \cap P_{L_{0}^{\varepsilon} v}^{\varepsilon}, P_{v}^{\varepsilon} \cap P_{L_{1}^{\varepsilon} v}^{\varepsilon}$, respectively, it maps $c_{1}$ onto the dual circle of the triangular interstice. Consequently, $Z_{0}^{\varepsilon}(v)$ maps the circle $c_{2}$ passing through $\varepsilon$ and $\varepsilon \omega^{4}$ which is orthogonal to $c_{1}$ onto $\partial P_{v}^{\varepsilon}$. Let $\varepsilon p_{2}$ be the center, and $\varepsilon \varrho_{2}$ be the radius of $c_{2}$. Then $p_{2}$ and $\varrho_{2}$ are constants.

How can we find a formula for $f^{\varepsilon}(v)$, the center of $P_{v}^{\varepsilon}$, in terms of $Z_{0}^{\varepsilon}(v)$ ? The inversion of $f^{\varepsilon}(v)$ in $\partial P_{v}^{\varepsilon}$ is $\infty$, obviously. The preimage of that inversion under $Z_{0}^{\varepsilon}(v)$ is the pole of $Z_{0}^{\varepsilon}(v)$. Let $q$ be the inversion in $c_{2}$ of the pole of $Z_{0}^{\varepsilon}(v)$. Note that when a point $z_{2}$ is the image of a point $z_{1}$ under an inversion in a circle $c$, then any Möbius transformtion $m$ will take $z_{2}$ to the inversion of $m\left(z_{1}\right)$ in the circle $m(c)$. Consequently, $Z_{0}^{\varepsilon}(v)(q)=f^{\varepsilon}(v)$. The pole of $Z_{0}^{\varepsilon}(v)$ is just the point $-d^{\varepsilon}(v) / c^{\varepsilon}(v)$. This gives

$$
q=\varepsilon p_{2}+\frac{\varepsilon^{2} \varrho_{2}^{2}}{-\varepsilon \overline{p_{2}}-\overline{d^{\varepsilon}(v)} / \overline{c^{\varepsilon}(v)}}=\varepsilon p_{2}+\frac{\varepsilon^{2} \varrho_{2}^{2} \overline{c^{\varepsilon}(v)}}{-\varepsilon \overline{c^{\varepsilon}(v) p_{2}}-\overline{d^{\varepsilon}(v)}}
$$

It is therefore clear from Lemma 2.2 that $f^{\varepsilon}(v)=Z_{0}^{\varepsilon}(v)(q)$ converges $C^{\infty}$ to $f$. 
Note that

$$
r^{\varepsilon}(w)+r^{\varepsilon}\left(L_{k}^{\varepsilon} w\right)=\left|f^{\varepsilon}(w)-f^{\varepsilon}\left(L_{k}^{\varepsilon} w\right)\right|=\varepsilon\left|\partial_{k}^{\varepsilon} f^{\varepsilon}(v)\right| .
$$

The three instances of this relation, $(w=v, k=0),(w=v, k=1)$ and $\left(w=L_{0}^{\varepsilon} v, k=2\right)$, give

$$
r^{\varepsilon}(v)=\frac{1}{2} \varepsilon\left(\left|\partial_{0}^{\varepsilon} f^{\varepsilon}(v)\right|+\left|\partial_{1}^{\varepsilon} f^{\varepsilon}(v)\right|-\left|\partial_{2}^{\varepsilon} f^{\varepsilon}\left(L_{0} v\right)\right|\right) .
$$

Because $\partial_{0}^{\varepsilon} f^{\varepsilon}$ converges $C^{\infty}$ to $f^{\prime}$ it follows from Lemma 2.2 that $\left|\partial_{0}^{\varepsilon} f\right|$ converges $C^{\infty}$ to $\left|f^{\prime}\right|$. Similarly, $\left|\partial_{k}^{\varepsilon} f\right| \rightarrow\left|f^{\prime}\right|$. Consequently, $2 r^{\varepsilon} / \varepsilon$ converges $C^{\infty}$ to $\left|f^{\prime}\right|$.

\section{Remarks: More general combinatorics and disk patterns}

Consider a locally finite disk packing $R$ in the plane, which is invariant under two linearly independent translations, and assume that all the interstices are triangular. It is well known that one may use the rescaled packings $R^{\varepsilon}=\varepsilon R=\{\varepsilon D: D \in R\}, \varepsilon>0$, to construct approximations to conformal mappings, and that the derivatives up to the second order converge (see, e.g., [8] or [9]). We now discuss the definition and convergence of the Schwarzian in this general case.

We will use $G^{\varepsilon}$ to denote the embedded graph of the packing $\varepsilon R$, and denote by $\Omega^{\varepsilon}$ the domain which approximates $\Omega ; R_{\Omega}^{\varepsilon}$ the subpacking of $\varepsilon R$ contained in $\Omega ; G_{\Omega}^{\varepsilon}$ the graph of $R_{\Omega}^{\varepsilon}$; and $V_{\Omega}^{\varepsilon}$ the vertex set of $G_{\Omega}^{\varepsilon}$. Let $P^{\varepsilon}$ be the disk packing in $\bar{U}$ whose graph is equivalent to $G_{\Omega}^{\varepsilon}$, such that the "boundary" disks are all tangent to the unit circle $\partial U$. We may then define $f^{\varepsilon}$ to be the map which maps the centers of disks in $R_{\Omega}^{\varepsilon}$ to the centers of the corresponding disks in $P^{\varepsilon}$. The problem is, does $f^{\varepsilon}$ converge in $C^{\infty}$ ? The meaning of the convergence is that the restriction of $f^{\varepsilon}$ to the intersection of $V_{\Omega}^{\varepsilon}$ with any lattice converges.

For each interior edge $e$ in the graph $G_{\Omega}^{\varepsilon}$, we may define two Möbius invariants $s^{\prime}(e)$ and $s^{\prime \prime}(e)$, corresponding to the packings $R_{\Omega}^{\varepsilon}$ and $P^{\varepsilon}$, respectively. (The invariant $s^{\prime}(e)$ is obtained by comparing the configuration of the four circles in $R_{\Omega}^{\varepsilon}$ related to $e$ to a configuration of four circles related to an edge in the hexagonal combinatorics, and similarly for $s^{\prime \prime}(e)$.) Then the Schwarzian derivative on the edge may be defined by $h(e)=\varepsilon^{-2}\left(s^{\prime \prime}(e)-s^{\prime}(e)\right)$. The equations of $s^{\prime}(e)$ and $s^{\prime \prime}(e)$ can be derived similarly.

The proof of the uniform boundedness of $h(e)$ (Lemma 6.1) is almost identical in this case. In fact, the proof of [6] works even better in the general abstract setting (see [8] for the necessary modifications). However, except for a few nice cases, it seems difficult to derive the equation for the "Laplacian" of the Schwarzians from the equations of the Möbius invariants.

One may consider disk patterns instead of disk packings. In this case, $C^{0}$-convergence follows by a compactness argument using the rigidity theorem of [7] and the topological 
lemma of [9]. In the special case of the square grid pattern (or in short SG pattern), the Möbius invariants and their equations have been worked out in [16], as we remarked earlier. One may define the discrete Schwarzians and find a similar formula for their Laplacians as in $\S 5$ of the present paper. On the other hand, because the angles of intersection between pairs of disks in a square grid pattern are either 0 or $\frac{1}{2} \pi$, the inversions on the circles generate a Kleinian group. Thus the method of [6] can be extended, although some nontrivial modifications are needed. This implies the boundedness of the Schwarzians. So the technique of this paper does generalize to SG patterns. The general case is still open.

There is an alternative method, which has been successful in proving the rigidity of locally finite disk patterns in the plane (see [7]). Using a refinement of that argument, it is possible to study the logarithm of the ratio of radii of a pair of disk patterns, and show that they converge to the harmonic function $\log \left|f^{\prime}(z)\right|$, where $f(z)$ denotes the conformal mapping from $\Omega$ to $U$. We conjecture that the convergence is $C^{\infty}$. If true, it would then be an elementary matter to deduce the $C^{\infty}$-convergence of the discrete function which maps the center of a disk to the center of the corresponding disk.

\section{References}

[1] Aharonov, D., The hexagonal packing lemma and discrete potential theory. Canad. Math. Bull., 33 (1990), 247-252.

[2] - The hexagonal packing lemma and the Rodin Sullivan conjecture. Trans. Amer. Math. Soc., 343 (1994), 157-167.

[3] Bowers, P. L., The upper Perron method for labeled complexes with applications to circle packings. Math. Proc. Cambridge Philos. Soc., 114 (1993), 321-345.

[4] Colin De Verdière, Y., Un principe variationnel pour les empilements de cercles. Invent. Math., 104 (1991), 655-669.

[5] Doyle, P. G., He, Z.-X. \& Rodin, B., Second derivatives of circle packings and conformal mappings. Discrete Comput. Geom., 11 (1994), 35-49.

[6] HE, Z.-X., An estimate for hexagonal circle packings. J. Differential Geom., 33 (1991), 395-412.

[7] - Rigidity of infinite disk patterns. Preprint, 1996.

[8] He, Z.-X. \& Rodin, B., Convergence of circle packings of finite valence to Riemann mappings. Comm. Anal. Geom., 1 (1993), 31-41.

[9] He, Z.-X. \& Schramm, O., On the convergence of circle packings to the Riemann map. Invent. Math., 125 (1996), 285-305.

[10] Koebe, P., Kontaktprobleme der konformen Abbildung. Ber. Verh. Sächs. Akad. Wiss. Leipzig, Math.-Phys. Kl., 88 (1936), 141-164.

[11] LeHto, O., Univalent Functions and Teichmüller Spaces. Graduate Texts in Math., 109. Springer-Verlag, New York, 1987.

[12] Marden, A. \& Rodin, B., On Thurston's formulation and proof of Andreev's theorem, in Computational Methods and Function Theory (Valparaiso, 1989), pp. 103-115. Lecture Notes in Math., 1435. Springer-Verlag, Berlin, 1990. 
[13] Rodin, B., Schwarz's lemma for circle packings. Invent. Math., 89 (1987), 271-289.

[14] - Schwarz's lemma for circle packings, II. J. Differential Geom., 30 (1989), 539-554.

[15] Rodin, B. \& Sullivan, D., The convergence of circle packings to the Riemann mapping. J. Differential Geom., 26 (1987), 349-360.

[16] Schramm, O., Circle patterns with the combinatorics of the square grid. Duke Math. J., 86 (1997), 347-389.

[17] Stephenson, K., A probablistic proof of Thurston's conjecture on circle packings. Preprint.

[18] Thurston, W.P., The Geometry and Topology of 3-manifolds. Princeton Univ. Notes, Princeton, NJ, 1982.

[19] - The finite Riemann mapping theorem. Unpublished talk given at the International Symposium in Celebration of the Proof of the Bieberbach Conjecture (Purdue University, 1985).

ZHENG-Xu He

Department of Mathematics

University of California, San Diego

La Jolla, CA 92093-0112

U.S.A.

zhe@math.ucsd.edu

Received December 13, 1996

\section{OdED SCHRAMM}

Department of Mathematics

The Weizmann Institute of Science

Rehovot 76100

Israel

schramm@wisdom.weizmann.ac.il 